\title{
A Three-Dimensional Range Migration Algorithm for Downward-Looking 3D-SAR with Single-Transmitting and Multiple-Receiving Linear Array Antennas
}

\author{
Lei Du, ${ }^{1,2,3}$ Yanping Wang, ${ }^{1,2}$ Wen Hong, ${ }^{1,2}$ Weixian Tan, ${ }^{1,2}$ and Yirong Wu ${ }^{1,2}$ \\ ${ }^{1}$ The National Key Laboratory of Microwave Imaging Technology (MITL), P.O. Box 2702, Beijing 100190, China \\ ${ }^{2}$ Institute of Electronics, Chinese Academy of Sciences (IECAS), P.O. Box 2702, Beijing 100190, China \\ ${ }^{3}$ The Graduate University of Chinese Academy of Sciences (GUCAS), Beijing 100049, China
}

Correspondence should be addressed to Yanping Wang, ypwang@mail.ie.ac.cn

Received 14 June 2009; Revised 29 October 2009; Accepted 16 December 2009

Academic Editor: Andreas Reigber

Copyright ( 92010 Lei Du et al. This is an open access article distributed under the Creative Commons Attribution License, which permits unrestricted use, distribution, and reproduction in any medium, provided the original work is properly cited.

\begin{abstract}
This paper presents a three-dimensional (3D) range migration algorithm (RMA) suitable for downward-looking 3D-SAR with single-transmitting and multiple-receiving linear array antennas (STMR-LAA). As the round-trip range equation in 3D-SAR with STMR-LAA is a dual square root, the signal spectrum in 3D wavenumber domain contains nonlinear phase terms besides constant and linear phase terms. In this paper, the approximate expression of the signal spectrum is derived by expanding the implicit phase term to its Taylor series. Then the constant and nonlinear phase terms are calculated and compensated by multiplying the wavenumber filters. Finally, a 3D wavenumber mapping is proposed to make the signal evenly sampled in 3D wavenumber domain. Some simulating results are presented to validate the correctness of the analysis and the feasibility of the algorithm. In addition, the required accuracy on the platform position is analyzed at the end of the paper.
\end{abstract}

\section{Introduction}

Synthetic aperture radar (SAR) is a well-developed technique for remote sensing. It can provide two-dimensional (2D) high-resolution radar images along azimuth and slant-range directions. However, since 2D radar images are obtained by projecting the three-dimensional (3D) distributed targets onto the 2D plane, they usually suffer from geometric distortions, such as foreshortening, layover, and so forth. Furthermore, conventional SAR system generally works at the side-looking mode and exhibits strong shadowing caused by buildings, hills, and valleys, which may result in the information loss of the explored area [1].

To overcome the disadvantages mentioned above, the concept of 3D-SAR imaging with linear array antennas (LAA) was proposed [1-3]. 3D-SAR with LAA combines the theories of real and synthetic aperture and can provide high-resolution radar images in three dimensions: azimuth, ground-range, and elevation. In addition, 3D-SAR with LAA can alleviate shadowing effects by looking downwards.

Due to its advanced performance, 3D-SAR with LAA has attracted wide attention. Two airborne downwardlooking 3D-SAR with LAA systems, DRIVE [4-7] and ARTINO [8-16], are being developed at ONERA and FGANFHR, respectively. The LAA used in these two systems are bistatic configuration $[3,10]$, which means that some antenna elements (or single antenna element) are used for transmitting and the others are used for receiving. As the round-trip range equation in bistatic configuration is composed of dual square roots, the imaging turns out to be more difficult than that in monostatic configuration. Giret et al. [3] made some simplifications to the dual square roots with Fresnel approximation and proposed an algorithm derived from monostatic imaging algorithm. A shortcoming 
of the algorithm is the range error caused by Fresnel approximation. Klare et al. $[8,9]$ introduced the concept of virtual antenna elements formed by the mean positions of every single transmitting and receiving antenna element to transform bistatic configuration into monostatic configuration and proposed a $3 \mathrm{D}$ imaging algorithm for ARTINO with beamforming operation in cross-track direction. However, the positions of virtual antenna elements depend on target locations, especially for the near-field targets.

Meanwhile, Reigber [17] proposed a 3D range migration algorithm (RMA) for SAR tomography imaging. LopezSanchez and Fortuny [18] and Fortuny [19] developed a 3D RMA for 2D planar scanning aperture in the near-field zone of the target. Tan et al. [20] proposed a 3D RMA for SAR tomography imaging with digital spotlight in the elevation direction. All of these 3D RMAs are suitable for the monostatic configuration, where the signal spectrum in $3 \mathrm{D}$ wavenumber domain only contains constant and linear phase terms. However, due to the dual square root in the bistatic configuration, the signal spectrum in $3 \mathrm{D}$ wavenumber domain contains nonlinear phase terms besides constant and linear phase terms. Therefore, the 3D RMAs [17-20] mentioned above cannot be applied to the bistatic configuration directly.

In this paper, we propose a 3D RMA which can be applied to downward-looking 3D-SAR with singletransmitting and multiple-receiving linear array antennas (STMR-LAA). Firstly, we derive the approximate expression of the signal spectrum by expanding the implicit phase term to its Taylor series, where quartic and higher-order phase terms are neglected because the phase error caused by these phase terms contributes little to the analysis. Secondly, after the removal of constant phase term, quadratic and cubic phase terms caused by the bistatic configuration are calculated and compensated by multiplying a wavenumber filter, where some approximations are considered to improve computational efficiency. Finally, a 3D wavenumber mapping is proposed according to the expression of the residual phase terms, and an interpolation is performed to make the signal evenly sampled in $3 \mathrm{D}$ wavenumber domain.

The paper is organized as follows. The imaging geometry is described in Section 2. The signal properties are analyzed, and a 3D RMA for downward-looking 3D-SAR with STMRLAA is derived in Section 3, where the removal of constant phase term, the compensation of nonlinear phase terms, and the mapping and interpolation in 3D wavenumber domain are highlighted. Simulation results are shown in Section 4. The required accuracy on the platform position to generate the 3D images is discussed in Section 5, and Section 6 gives conclusions of this paper.

\section{Imaging Geometry}

We consider the simplified imaging scenario in a 3D spatial domain $O X Y Z$, and the coordinate axes are defined in Figure 1. The platform is supposed to fly at the altitude $H$

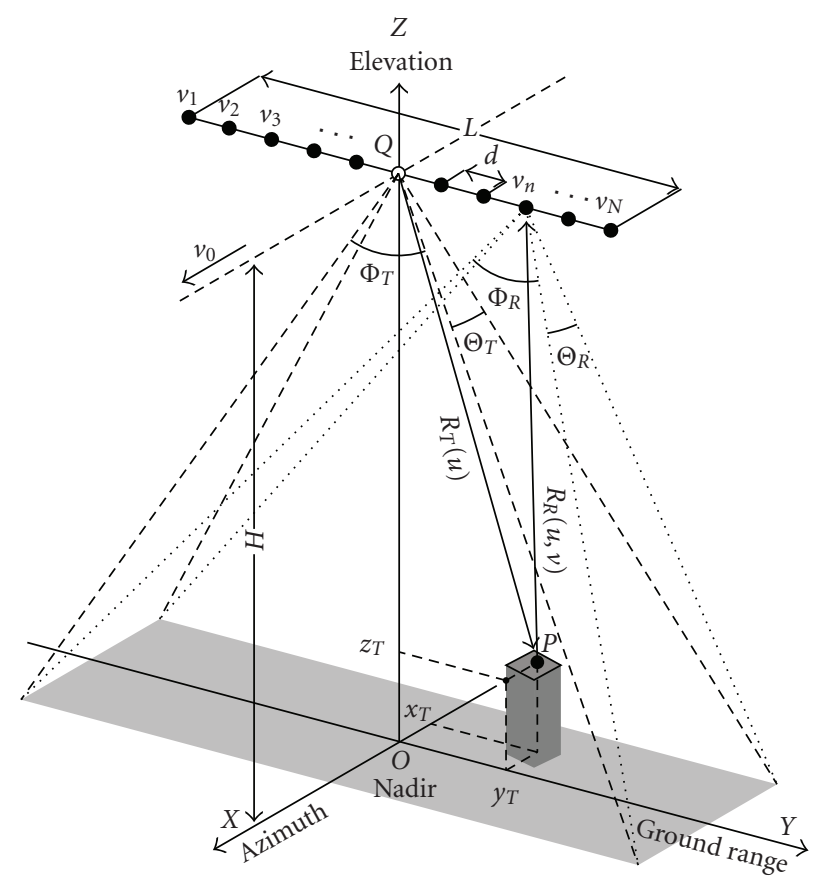

- Receive antenna element

o Transmit antenna

FIGURE 1: Imaging geometry of downward-looking 3D-SAR with STMR-LAA, where $x$-axis, $y$-axis and $z$-axis denote the azimuth (along-track), ground-range (cross-track), and elevation directions respectively, and $O$ indicates the origin of the Cartesian coordinates.

along the $x$-axis with the constant velocity $v_{0}$. The illuminated scenario is located at the nadir area of the platform, and the origin of the coordinate system is located at the nadir point.

The STMR-LAAs of the 3D-SAR are composed of a transmitting antenna element $(\mathrm{Tx})$ and $N$ receiving antenna elements $(\mathrm{Rx})$ and distributed along the cross-track direction. Tx is located at the center of the LAA (i.e., $Q$ in Figure 1), and its position is $\{u, 0, H\}$, where $u=v_{0} t_{m}$, and $t_{m}$ indicates the slow time. The entire Rxs are evenly spaced with distance $d$. Therefore, the length of LAA $L$ equals to $(N-1) d$, and the position of the $n$th $\operatorname{Rx}$ is $\{u, v, H\}$, where $v=-L / 2+(n-1) d$ and $n=1,2,3, \ldots, N$.

Let a point target $P$ locate at $\left\{x_{T}, y_{T}, z_{T}\right\}$ with reflectivity $\sigma\left(x_{T}, y_{T}, z_{T}\right)$. The distance from Tx to the target $P$ is given by

$$
R_{T}(u)=\sqrt{\left(u-x_{T}\right)^{2}+y_{T}^{2}+\left(H-z_{T}\right)^{2}}
$$

shown in Figure 1, and the distance from $P$ to the $n$th $\mathrm{Rx}$ is

$$
R_{R}(u, v)=\sqrt{\left(u-x_{T}\right)^{2}+\left(v-y_{T}\right)^{2}+\left(H-z_{T}\right)^{2}} .
$$

Therefore, the round-trip range equation can be written as

$$
R(u, v)=R_{T}(u)+R_{R}(u, v)
$$




\section{Formulation of the 3D RMA}

Here, we use the geometric relations and symbols illustrated in Figure 1 and assume that the transmitted signal $p(t)$ is a chirp with the bandwidth $B$ :

$$
p(t)=\operatorname{rect}\left(\frac{t}{T}\right) \exp \left\{j 2 \pi\left(f_{c} t+\frac{1}{2} \mu t^{2}\right)\right\}
$$

where $t$ denotes the fast time; $T$ indicates the pulse duration; $f_{c}$ is the carrier frequency; $\mu$ is the chirp rate; the transmitted signal bandwidth $B=\mu T$; $\operatorname{rect}(\cdot)$ is a rectangular window function, which means $\operatorname{rect}(t / T)=1$ for $|t| \leq T / 2$, and zero otherwise.

3.1. Signal Spectrum in 3D Wavenumber Domain. After coherent demodulation, the received signal can be written as

$$
\begin{aligned}
s_{0}(t, u, v)=\iiint & \sigma\left(x_{T}, y_{T}, z_{T}\right) \operatorname{rect}\left\{\left[t-\frac{R(u, v)}{c}\right] / T\right\} \\
& \times \exp \left\{j \pi \mu\left(t-\frac{R(u, v)}{c}\right)^{2}\right\} \\
& \times \exp \left\{-j 2 \pi f_{c} \frac{R(u, v)}{c}\right\} d x_{T} d y_{T} d z_{T}
\end{aligned}
$$

where $c$ is the speed of light. The antenna pattern and its type are not considered here since they contribute nothing to the essence of the analyzed themes.

The Fourier transform of the received signal $s_{0}(t, u, v)$ with respect to the fast-time $t$ is

$$
\begin{aligned}
S_{0}\left(f_{t}, u, v\right)=\iiint & \sigma\left(x_{T}, y_{T}, z_{T}\right) \operatorname{rect}\left(\frac{f_{t}}{\mu T}\right) \exp \left\{-j \pi \frac{f_{t}^{2}}{\mu}\right\} \\
& \times \exp \left\{-j 2 \pi f_{t} \frac{R(u, v)}{c}\right\} \\
& \times \exp \left\{-j 2 \pi f_{c} \frac{R(u, v)}{c}\right\} d x_{T} d y_{T} d z_{T}
\end{aligned}
$$

where $f_{t}$ is the slant-range frequency domain for $t$, and $\left|f_{t}\right| \leq$ $B / 2$.

The slant-range compression is performed via the slantrange frequency domain matched filter:

$$
H_{1}\left(f_{t}\right)=\operatorname{rect}\left(\frac{f_{t}}{\mu T}\right) \exp \left\{j \pi \frac{f_{t}^{2}}{\mu}\right\} .
$$

After slant-range compression, the signal can be written as

$$
\begin{aligned}
S_{1}\left(f_{t}, u, v\right)= & S_{0}\left(f_{t}, u, v\right) \times H_{1}\left(f_{t}\right) \\
= & \iiint \sigma\left(x_{T}, y_{T}, z_{T}\right) \\
& \quad \times \exp \left\{-j 2 \pi \frac{f_{t}+f_{c}}{c} R(u, v)\right\} d x_{T} d y_{T} d z_{T} .
\end{aligned}
$$

Let $k=2 \pi\left(f_{t}+f_{c}\right) / c$ denote the wavenumber domain of the transmitted signal, and $k \in\left[k_{\min }, k_{\max }\right]$, where $k_{\min }=$ $2 \pi\left(f_{c}-B / 2\right) / c, k_{\max }=2 \pi\left(f_{c}+B / 2\right) / c$ and $k_{c}=2 \pi f_{c} / c$; then (8) can be rewritten as

$$
S_{1}(k, u, v)=\iiint \sigma\left(x_{T}, y_{T}, z_{T}\right) \exp \{-j k R(u, v)\} d x_{T} d y_{T} d z_{T}
$$

in the slant-range wavenumber domain.

The 2D Fourier transform of the signal $S_{1}(k, u, v)$ with respect to $(u, v)$ is

$$
\begin{aligned}
& S_{2}\left(k, k_{u}, k_{v}\right) \\
& =\iint S_{1}(k, u, v) \exp \left\{-j\left(k_{u} u+k_{v} v\right)\right\} d u d v \\
& =\iiint \sigma\left(x_{T}, y_{T}, z_{T}\right) \\
& \quad \times\left\{\iint \exp \left\{-j\left[k R(u, v)+k_{u} u+k_{v} v\right]\right\} d u d v\right\} d x_{T} d y_{T} d z_{T},
\end{aligned}
$$

where $k_{u}$ is the azimuth wavenumber domain for $u$, and $k_{v}$ is the cross-track wavenumber domain for $v$. Let $U=u-x_{T}$ and $V=v-y_{T}$, then (10) can be rewritten as

$$
\begin{aligned}
& S_{3}\left(k, k_{u}, k_{v}\right) \\
& =\iiint \sigma\left(x_{T}, y_{T}, z_{T}\right) \exp \left\{-j\left(k_{u} x_{T}+k_{v} y_{T}\right)\right\} \\
& \times\left\{\iint \exp \left\{-j\left[k R(U, V)+k_{u} U+k_{v} V\right]\right\} d U d V\right\} d x_{T} d y_{T} d z_{T},
\end{aligned}
$$

where $R(U, V)=\sqrt{U^{2}+y_{T}^{2}+\left(H-z_{T}\right)^{2}}+\sqrt{U^{2}+V^{2}+\left(H-z_{T}\right)^{2}}$.

The principle of stationary phase (POSP) can be applied to solve the inner integral of (11). Here, we use $\Phi(U, V)$ to denote the negative phase term in (11), that is,

$$
\Phi(U, V)=k R(U, V)+k_{u} U+k_{v} V
$$

Based on the POSP, the stationary points $U_{0}$ and $V_{0}$ should satisfy the equations set:

$$
\begin{aligned}
& \left.\frac{\partial \Phi(U, V)}{\partial U}\right|_{U=U_{0}, V=V_{0}}=0, \\
& \left.\frac{\partial \Phi(U, V)}{\partial V}\right|_{U=U_{0}, V=V_{0}}=0 .
\end{aligned}
$$


According to the definition of $\Phi(U, V)$ in (12), (13) can be written as

$$
\begin{aligned}
& k\left[\frac{U_{0}}{\sqrt{U_{0}^{2}+y_{T}^{2}+\left(H-z_{T}\right)^{2}}}+\frac{U_{0}}{\sqrt{U_{0}^{2}+V_{0}^{2}+\left(H-z_{T}\right)^{2}}}\right] \\
& \quad+k_{u}=0, \\
& k \frac{V_{0}}{\sqrt{U_{0}^{2}+V_{0}^{2}+\left(H-z_{T}\right)^{2}}}+k_{v}=0 .
\end{aligned}
$$

It is too complicated to obtain the analytic solutions of equations set (14), and so we cannot have the explicit expressions of the stationary points $U_{0}$ and $V_{0}$. However, both $U_{0}$ and $V_{0}$ are the function of $k, k_{u}, k_{v}, y_{T}$, and $z_{T}$, that is, $U_{0}=U_{0}\left(k, k_{u}, k_{v} ; y_{T}, z_{T}\right)$ and $V_{0}=V_{0}\left(k, k_{u}, k_{v} ; y_{T}, z_{T}\right)$. Therefore, the implicit expression of the signal spectrum in $3 \mathrm{D}$ wavenumber domain can be written as

$$
\begin{aligned}
& S_{3}\left(k, k_{u}, k_{v}\right) \\
& =\iiint \sigma\left(x_{T}, y_{T}, z_{T}\right) \\
& \quad \times \exp \left\{-j\left(k_{u} x_{T}+k_{v} y_{T}+\Phi\left(U_{0}, V_{0}\right)\right)\right\} d x_{T} d y_{T} d z_{T},
\end{aligned}
$$

where unessential multiplicative complex constants are not represented in the formula, and

$$
\Phi\left(U_{0}, V_{0}\right)=k R\left(U_{0}, V_{0}\right)+k_{u} U_{0}+k_{v} V_{0} .
$$

$\Phi\left(U_{0}, V_{0}\right)$ is written as $\Phi_{0}$ for notational simplicity hereafter.

From (16), we can see that $\Phi_{0}$ is the function of $k, k_{u}, k_{v}$, $y_{T}$, and $z_{T}$. If $\Phi_{0}$ can be factorized to a combination of linear phase terms with respect to $y_{T}$ and $z_{T}$, that is,

$$
\Phi_{0}=k_{1}\left(k, k_{u}, k_{v}\right) y_{T}+k_{2}\left(k, k_{u}, k_{v}\right) z_{T}
$$

then (15) can be written as

$$
\begin{aligned}
& S_{4}\left(k_{x}, k_{y}, k_{z}\right) \\
& =\iiint \sigma\left(x_{T}, y_{T}, z_{T}\right) \\
& \quad \times \exp \left\{-j\left(k_{x} x_{T}+k_{y} y_{T}+k_{z} z_{T}\right)\right\} d x_{T} d y_{T} d z_{T},
\end{aligned}
$$

where

$$
\begin{gathered}
k_{x}=k_{u}, \\
k_{y}=k_{v}+k_{1}\left(k, k_{u}, k_{v}\right), \\
k_{z}=k_{2}\left(k, k_{u}, k_{v}\right) .
\end{gathered}
$$

After the wavenumber interpolation and 3D IFFT operation, the $3 \mathrm{D}$ image in the spatial domain $O X Y Z$ can be generated. However, unlike the monostatic case [17-20], the $\Phi_{0}$ in (16) contains nonlinear phase terms besides constant and linear phase ones. Since precise 3D RMA does not exist in 3DSAR with STMR-LAA, only an approximate solution can be found.

In this paper, we expand $\Phi_{0}$ to its Taylor series and compensate the phase terms except for the linear phase ones. The terms left in $\Phi_{0}$ can be expressed as (17), and the following steps are the same as the monostatic case. Expanding $\Phi_{0}$ to its Taylor series at $y_{T}=0, z_{T}=0$ and neglecting the quartic and higher-order terms, we have

$$
\begin{aligned}
\Phi_{0} \approx & \Phi_{\text {const }}+\left.\left(y_{T} \frac{\partial}{\partial y_{T}}+z_{T} \frac{\partial}{\partial z_{T}}\right) \Phi_{0}\right|_{y_{T}=0, z_{T}=0} \\
& +\left.\frac{1}{2}\left(y_{T} \frac{\partial}{\partial y_{T}}+z_{T} \frac{\partial}{\partial z_{T}}\right)^{2} \Phi_{0}\right|_{y_{T}=0, z_{T}=0} \\
& +\left.\frac{1}{6}\left(y_{T} \frac{\partial}{\partial y_{T}}+z_{T} \frac{\partial}{\partial z_{T}}\right)^{3} \Phi_{0}\right|_{y_{T}=0, z_{T}=0,}
\end{aligned}
$$

where the first term is constant and independent of the target location; the second term is the linear phase term, which contributes to focusing; the third and forth terms are the main nonlinear phase terms, which will cause defocusing without compensation. The effect of every term in (20) will be analyzed in detail as follows.

3.2. Analysis and Removal of Constant Phase Term. The constant phase term $\Phi_{\text {const }}$ in (20) can be written as

$$
\begin{aligned}
\Phi_{\text {const }} & =\Phi_{0}\left(k, k_{u}, k_{v} ; y_{T}=0, z_{T}=0\right) \\
& =k R\left(U_{00}, V_{00}\right)+k_{u} U_{00}+k_{v} V_{00},
\end{aligned}
$$

where $U_{00}=U_{0}\left(k, k_{u}, k_{v} ; y_{T}=0, z_{T}=0\right), V_{00}=V_{0}\left(k, k_{u}\right.$, $\left.k_{v} ; y_{T}=0, z_{T}=0\right)$, and

$$
R\left(U_{00}, V_{00}\right)=\sqrt{U_{00}^{2}+H^{2}}+\sqrt{U_{00}^{2}+V_{00}^{2}+H^{2}} .
$$

Since $U_{00}$ and $V_{00}$ are the special cases of $U_{0}$ and $V_{0}$ at $y_{T}=0, z_{T}=0$, they can be considered as the particular solutions of equations set (14). Thus, we have

$$
\begin{gathered}
k\left(\frac{U_{00}}{\sqrt{U_{00}^{2}+H^{2}}}+\frac{U_{00}}{\sqrt{U_{00}^{2}+V_{00}^{2}+H^{2}}}\right)+k_{u}=0, \\
k \frac{V_{00}}{\sqrt{U_{00}^{2}+V_{00}^{2}+H^{2}}}+k_{v}=0 .
\end{gathered}
$$

The analytic solutions of equations set (23) are

$$
\begin{gathered}
U_{00}=-\frac{k_{u} H}{\sqrt{\left(k+\sqrt{k^{2}-k_{v}^{2}}\right)^{2}-k_{u}^{2}}}, \\
V_{00}=-\frac{k_{v}}{\sqrt{k^{2}-k_{v}^{2}}} \frac{\left(k+\sqrt{k^{2}-k_{v}^{2}}\right) H}{\sqrt{\left(k+\sqrt{k^{2}-k_{v}^{2}}\right)^{2}-k_{u}^{2}}} .
\end{gathered}
$$


Thus $\Phi_{\text {const }}$ is equal to

$$
\Phi_{\text {const }}=H \sqrt{\left(k+\sqrt{k^{2}-k_{v}^{2}}\right)^{2}-k_{u}^{2}} .
$$

The removal of constant phase term can be performed by multiplying the following filter in 3D wavenumber domain:

$$
H_{2}\left(k, k_{u}, k_{v}\right)=\exp \left\{j H \sqrt{\left(k+\sqrt{k^{2}-k_{v}^{2}}\right)^{2}-k_{u}^{2}}\right\} .
$$

3.3. Analysis and Compensation of Nonlinear Phase Terms. The nonlinear phase terms do not exist in the monostatic case but emerge here due to the bistatic configuration of LAA. Thus, the compensation of nonlinear phase terms is the key point of the 3D RMA proposed in this paper. According to (20), the nonlinear phase terms $\Phi_{\text {nonline }}$ in $\Phi_{0}$ mainly include two parts, that is,

$$
\Phi_{\text {nonline }}=\Phi_{\text {quad }}+\Phi_{\text {cubic }}
$$

where $\Phi_{\text {quad }}$ denotes the quadratic phase term,

$$
\begin{aligned}
\Phi_{\text {quad }}= & \left.\frac{1}{2}\left(y_{T} \frac{\partial}{\partial y_{T}}+z_{T} \frac{\partial}{\partial z_{T}}\right)^{2} \Phi_{0}\right|_{y_{T}=0, z_{T}=0} \\
= & \frac{1}{2}\left[\left.\frac{\partial^{2} \Phi_{0}}{\partial y_{T}^{2}}\right|_{y_{T}=0, z_{T}=0} y_{T}^{2}\right. \\
& \left.+\left.2 \frac{\partial^{2} \Phi_{0}}{\partial y_{T} \partial z_{T}}\right|_{y_{T}=0, z_{T}=0} y_{T} z_{T}+\left.\frac{\partial^{2} \Phi_{0}}{\partial z_{T}^{2}}\right|_{y_{T}=0, z_{T}=0} z_{T}^{2}\right],
\end{aligned}
$$

and $\Phi_{\text {cubic }}$ denotes the cubic phase term,

$$
\begin{aligned}
\Phi_{\text {cubic }}= & \left.\frac{1}{6}\left(y_{T} \frac{\partial}{\partial y_{T}}+z_{T} \frac{\partial}{\partial z_{T}}\right)^{3} \Phi_{0}\right|_{y_{T}=0, z_{T}=0} \\
= & \frac{1}{6}\left[\left.\frac{\partial^{3} \Phi_{0}}{\partial y_{T}^{3}}\right|_{y_{T}=0, z_{T}=0} y_{T}^{3}+\left.3 \frac{\partial^{3} \Phi_{0}}{\partial y_{T}^{2} \partial z_{T}}\right|_{y_{T}=0, z_{T}=0} y_{T}^{2} z_{T}\right. \\
& \left.+\left.3 \frac{\partial^{3} \Phi_{0}}{\partial y_{T} \partial z_{T}^{2}}\right|_{y_{T}=0, z_{T}=0} y_{T} z_{T}^{2}+\left.\frac{\partial^{3} \Phi_{0}}{\partial z_{T}^{3}}\right|_{y_{T}=0, z_{T}=0} z_{T}^{3}\right] .
\end{aligned}
$$

From (A.7) and (A.8) in Appendix A, the coefficients of $\Phi_{\text {quad }}$ associated with $y_{T}^{2}, y_{T} z_{T}$, and $z_{T}^{2}$ in (28) can be, respectively, calculated as follows:

$$
\begin{aligned}
& \left.\frac{\partial^{2} \Phi_{0}}{\partial y_{T}^{2}}\right|_{y_{T}=0, z_{T}=0} \\
& =k\left\{\frac{1}{\sqrt{U_{0}^{2}+y_{T}^{2}+\left(H-z_{T}\right)^{2}}}-\frac{y_{T}^{2}}{\left[U_{0}^{2}+y_{T}^{2}+\left(H-z_{T}\right)^{2}\right]^{3 / 2}}\right. \\
& \left.-\frac{U_{0} y_{T}}{\left[U_{0}^{2}+y_{T}^{2}+\left(H-z_{T}\right)^{2}\right]^{3 / 2}} \frac{\partial U_{0}}{\partial y_{T}}\right\}\left.\right|_{y_{T}=0, z_{T}=0} \\
& =\frac{k \sqrt{\left(k+\sqrt{k^{2}-k_{v}^{2}}\right)^{2}-k_{u}^{2}}}{H\left(k+\sqrt{k^{2}-k_{v}^{2}}\right)}, \\
& \left.\frac{\partial^{2} \Phi_{0}}{\partial y_{T} \partial z_{T}}\right|_{y_{T}=0, z_{T}=0} \\
& =k y_{T}\left\{\frac{H-z_{T}}{\left[U_{0}^{2}+y_{T}^{2}+\left(H-z_{T}\right)^{2}\right]^{3 / 2}}\right. \\
& \left.-\frac{U_{0}}{\left[U_{0}^{2}+y_{T}^{2}+\left(H-z_{T}\right)^{2}\right]^{3 / 2}} \frac{\partial U_{0}}{\partial z_{T}}\right\}\left.\right|_{y_{T}=0, z_{T}=0} \\
& =0 \text {, } \\
& \left.\frac{\partial^{2} \Phi_{0}}{\partial z_{T}^{2}}\right|_{y_{T}=0, z_{T}=0} \\
& =\left.\left\{-\frac{k_{u}}{U_{0}}-\frac{k_{u}\left(H-z_{T}\right)}{U_{0}^{2}} \frac{\partial U_{0}}{\partial z_{T}}\right\}\right|_{y_{T}=0, z_{T}=0} \\
& =-\frac{k_{u}}{U_{00}}-\frac{k_{u} H}{U_{00}^{2}} \times\left.\frac{\partial U_{0}}{\partial z_{T}}\right|_{y_{T}=0, z_{T}=0}=0,
\end{aligned}
$$

where $\partial U_{0} /\left.\partial z_{T}\right|_{y_{T}=0, z_{T}=0}=-U_{00} / H$ in (32) (see Appendix B). According to (30)-(32), there is only one term left in the quadratic phase term $\Phi_{\text {quad }}$, that is,

$$
\Phi_{\mathrm{quad}}=\frac{k \sqrt{1-q}}{2 H} y_{T}^{2}
$$

where

$$
q=\frac{k_{u}^{2}}{\left(k+\sqrt{k^{2}-k_{v}^{2}}\right)^{2}} .
$$

We can conclude that only the ground-range phase error term is included in $\Phi_{\text {quad }}$, which will cause defocusing in the ground-range direction without compensation. 
From (30)-(32), the coefficients of $\Phi_{\text {cubic }}$ associated with $y_{T}^{3}, z_{T}^{3}, y_{T} z_{T}^{2}$, and $y_{T}^{2} z_{T}$ in (29) can be, respectively, calculated as follows:

$$
\left.\frac{\partial^{3} \Phi_{0}}{\partial y_{T}^{3}}\right|_{y_{T}=0, z_{T}=0}=-\frac{2 U_{00}}{\left(\sqrt{U_{00}^{2}+H^{2}}\right)^{3}} \times\left.\frac{\partial U_{0}}{\partial y_{T}}\right|_{y_{T}=0, z_{T}=0}=0
$$

$$
\begin{gathered}
\left.\frac{\partial^{3} \Phi_{0}}{\partial z_{T}^{3}}\right|_{y_{T}=0, z_{T}=0}=-\frac{k_{u} H}{U_{00}^{2}} \times\left.\frac{\partial^{2} U_{0}}{\partial z_{T}^{2}}\right|_{y_{T}=0, z_{T}=0}=0 \\
\left.\frac{\partial^{3} \Phi_{0}}{\partial y_{T} \partial z_{T}^{2}}\right|_{y_{T}=0, z_{T}=0}=0
\end{gathered}
$$

$$
\begin{aligned}
& \left.\frac{\partial^{3} \Phi_{0}}{\partial y_{T}^{2} \partial z_{T}}\right|_{y_{T}=0, z_{T}=0} \\
& =\frac{k}{H \sqrt{U_{00}^{2}+H^{2}}}=\frac{k}{H^{2}} \sqrt{1-\frac{k_{u}^{2}}{\left(k+\sqrt{k^{2}-k_{v}^{2}}\right)^{2}}},
\end{aligned}
$$

where $\partial U_{0} /\left.\partial y_{T}\right|_{y_{T}=0, z_{T}=0}=0$ in (35) (see Appendix B), and $\partial^{2} U_{0} /\left.\partial z_{T}^{2}\right|_{y_{T}=0, z_{T}=0}=0$ in (36) (see Appendix C). According to (35)-(38), there is only one term left in $\Phi_{\text {cubic }}$, that is,

$$
\Phi_{\text {cubic }}=\frac{k \sqrt{1-q}}{2 H^{2}} y_{T}^{2} z_{T}
$$

Thus, the nonlinear phase terms $\Phi_{\text {nonline in }} \Phi_{0}$ can be written as

$$
\Phi_{\text {nonline }}=\frac{k \sqrt{1-q}}{2 H^{2}} y_{T}^{2}\left(H+z_{T}\right)
$$

The compensation of $\Phi_{\text {nonline }}$ can be performed by multiplying the following filter in $3 \mathrm{D}$ wavenumber domain (i.e., $\left(k, k_{u}, k_{v}\right)$ domain):

$$
H_{3}\left(k, k_{u}, k_{v} ; y_{T}, z_{T}\right)=\exp \left\{j \Phi_{\text {nonline }}\right\} .
$$

From (34), (40), and (41), we know that the compensation of $\Phi_{\text {nonline }}$ needs to take 5 variables into account, which are the slant-range wavenumber $k$, azimuth wavenumber $k_{u}$, cross-track wavenumber $k_{v}$, the location of target in the ground-range direction $y_{T}$, and elevation direction $z_{T}$. For the compensation of the nonlinear phase terms will require high computation time, some approximations will be considered to improve computational efficiency.

According to (9), the azimuth instantaneous wavenumber $k_{u}$ is equal to

$$
\begin{aligned}
k_{u} & =\frac{\partial}{\partial u}[-k R(u, v)] \\
& =-k\left[\sin \theta_{T}\left(x_{T}, y_{T}, z_{T}\right)+\sin \theta_{R n}\left(x_{T}, y_{T}, z_{T}\right)\right] .
\end{aligned}
$$

In (42), $\theta_{T}\left(x_{T}, y_{T}, z_{T}\right)=\arcsin \left\{\left(u-x_{T}\right) / R_{T}(u)\right\}$, which is the azimuth instantaneous aspect angle of the Tx to the point target $P$, and $\left|\theta_{T}\left(x_{T}, y_{T}, z_{T}\right)\right| \leq \Theta_{T} / 2$, where $\Theta_{T}$ is the azimuth beamwidth of the Tx. $\theta_{R n}\left(x_{T}, y_{T}, z_{T}\right)=\arcsin \{(u-$ $\left.\left.x_{T}\right) / R_{R}(u, v)\right\}$, which is the azimuth instantaneous aspect angle of the $n$th $\mathrm{Rx}$ to $P$, and $\left|\theta_{R n}\left(x_{T}, y_{T}, z_{T}\right)\right| \leq \Theta_{R} / 2$, where $\Theta_{R}$ is the azimuth beamwidth of the $\mathrm{Rx}$. Here, it is assumed that every Rx has the same azimuth beamwidth. Therefore, the maximum of $k_{u}$ for a fixed slant-range wavenumber $k$ is

$$
k_{u \max }=k\left[\sin \left(\frac{\Theta_{T}}{2}\right)+\sin \left(\frac{\Theta_{R}}{2}\right)\right] .
$$

Similarly, the cross-track instantaneous wavenumber $k_{v}$ is equal to

$$
k_{v}=\frac{\partial}{\partial v}[-k R(u, v)]=-k \sin \phi_{R n}\left(x_{T}, y_{T}, z_{T}\right) .
$$

In $(44), \phi_{R n}\left(x_{T}, y_{T}, z_{T}\right)=\arcsin \left\{\left(v-y_{T}\right) / R_{R}(u, v)\right\}$, which is the cross-track instantaneous aspect angle of the $n$th $\mathrm{Rx}$ to $P$, and $\left|\phi_{R n}\left(x_{T}, y_{T}, z_{T}\right)\right| \leq \Phi_{R} / 2$, where $\Phi_{R}$ is the cross-track beamwidth of the $\mathrm{Rx}$ and every $\mathrm{Rx}$ is assumed to have the same cross-track beamwidth. The maximum of $k_{v}$ for a fixed slant-range wavenumber $k$ is

$$
k_{v \max }=k \sin \left(\frac{\Phi_{R}}{2}\right) .
$$

According to (43) and (45), the maximum of $q$ is

$$
q_{\max }=\frac{k_{u \max }^{2}}{\left(k+\sqrt{k^{2}-k_{v \max }^{2}}\right)^{2}}=\left[\frac{\sin \left(\Theta_{T} / 2\right)+\sin \left(\Theta_{R} / 2\right)}{1+\cos \left(\Phi_{R} / 2\right)}\right]^{2} .
$$

If the parameters of 3D-SAR with STMR-LAA are chosen to satisfy $q_{\max } \ll 1$, then the wavenumber filter shown in (41) can be approximated as

$$
H_{3}\left(k ; y_{T}, z_{T}\right) \approx \exp \left\{j \frac{k}{2 H^{2}} y_{T}^{2}\left(H+z_{T}\right)\right\} .
$$

After approximation, the wavenumber filter only depends on $k, y_{T}$, and $z_{T}$, and the computational efficiency is improved. The nonlinear phase compensation can be performed by multiplying (47) in the slant-range wavenumber domain before the $2 \mathrm{D}$ Fourier transform with respect to $(u, v)$ (i.e., $(k, u, v)$ domain). The phase error between (41) and (47) will be discussed in Section 4.

3.4. Mapping and Interpolation in 3D Wavenumber Domain. After the removal of constant phase term $\Phi_{\text {const }}$ and compensation of nonlinear phase terms $\Phi_{\text {nonline }}$, the residual phase term in $\Phi_{0}$ can be written as

$$
\begin{aligned}
\Phi_{\text {line }} & =\left.\left(y_{T} \frac{\partial}{\partial y_{T}}+z_{T} \frac{\partial}{\partial z_{T}}\right) \Phi_{0}\right|_{y_{T}=0, z_{T}=0} \\
& =\left.\frac{\partial \Phi_{0}}{\partial y_{T}}\right|_{y_{T}=0, z_{T}=0} y_{T}+\left.\frac{\partial \Phi_{0}}{\partial z_{T}}\right|_{y_{T}=0, z_{T}=0} z_{T} \\
& =-z_{T} \sqrt{\left(k+\sqrt{k^{2}-k_{v}^{2}}\right)^{2}-k_{u}^{2}},
\end{aligned}
$$


where the coefficients of the phase terms with respect to $y_{T}$ and $z_{T}$ are derived in Appendix A. According to (48), the residual phase term in $\Phi_{0}$ is composed of the linear phase terms with respect to $y_{T}$ and $z_{T}$, and we can obtain that $k_{1}\left(k, k_{u}, k_{v}\right)=0$ and $k_{2}\left(k, k_{u}, k_{v}\right)=-\sqrt{\left(k+\sqrt{k^{2}-k_{v}^{2}}\right)^{2}-k_{u}^{2}}$ by comparing (48) with (17). Thus we can define a $3 \mathrm{D}$ wavenumber mapping:

$$
\begin{gathered}
k_{x}=k_{u}, \\
k_{y}=k_{v}, \\
k_{z}=-\sqrt{\left(k+\sqrt{k^{2}-k_{v}^{2}}\right)^{2}-k_{u}^{2}}
\end{gathered}
$$

which is used to transform the signal in the $\left(k, k_{u}, k_{v}\right)$ domain into the one in the $\left(k_{x}, k_{y}, k_{z}\right)$ domain. The 3D wavenumber mapping used here is distinguished from the one used in the monostatic case [17-20]. It essentially replaces wavenumber in the direction of wave propagation by wavenumber observed in the azimuth, ground-range, and elevation directions. The reason for the negative sign in (49c) is that the defined direction between wave propagation and the elevation is opposite in this paper.

After the 3D wavenumber mapping shown in ((49a), (49b), (49c)), the available discrete evenly spaced samples of $(t, u, v)$ are transformed into evenly spaced samples of $\left(k_{x}, k_{y}\right)$ and unevenly spaced samples of the elevation wavenumber $k_{z}$. Thus an interpolation shown in (49c) is performed to transform these unevenl $y$ spaced samples into evenly spaced ones in the $k_{z}$ domain.

Once the interpolation is done, we will obtain the expression shown in (18). After the 3D inverse Fourier transform of the signal $S_{4}\left(k_{x}, k_{y}, k_{z}\right)$ with respect to $\left(k_{x}, k_{y}, k_{z}\right)$, the reconstructed target image in a $3 \mathrm{D}$ spatial domain $O X Y Z$ can be expressed as

$$
\begin{aligned}
s_{4}(x, y, z)=\iiint & \sigma\left(x_{T}, y_{T}, z_{T}\right) \operatorname{sinc}\left\{\frac{B_{x_{T}}\left(x-x_{T}\right)}{2 \pi}\right\} \\
& \times \operatorname{sinc}\left\{\frac{B_{y_{T}}\left(y-y_{T}\right)}{2 \pi}\right\} \\
& \times \operatorname{sinc}\left\{\frac{B_{z_{T}}\left(z-z_{T}\right)}{2 \pi}\right\} d x_{T} d y_{T} d z_{T},
\end{aligned}
$$

where the sinc function is defined as $\operatorname{sinc}(a)=\sin (\pi a) /(\pi a)$; $B_{x_{T}}$ is the bandwidth of the target $P$ spectral support in the $k_{x}$ domain, which determines the azimuth resolution; $B_{y_{T}}$ is the bandwidth of the target $P$ spectral support in the $k_{y}$ domain, which determines the ground-range resolution; $B_{z_{T}}$ is the bandwidth of the target $P$ spectral support in the $k_{z}$ domain, which determines the elevation resolution.

3.5. Summary of 3D RMA. So far, the 3D RMA for 3DSAR with STMR-LAA has been deduced in 3D wavenumber domain. The block scheme of this algorithm is shown in Figure 2. The execution steps and some implementation details are explained as follows.

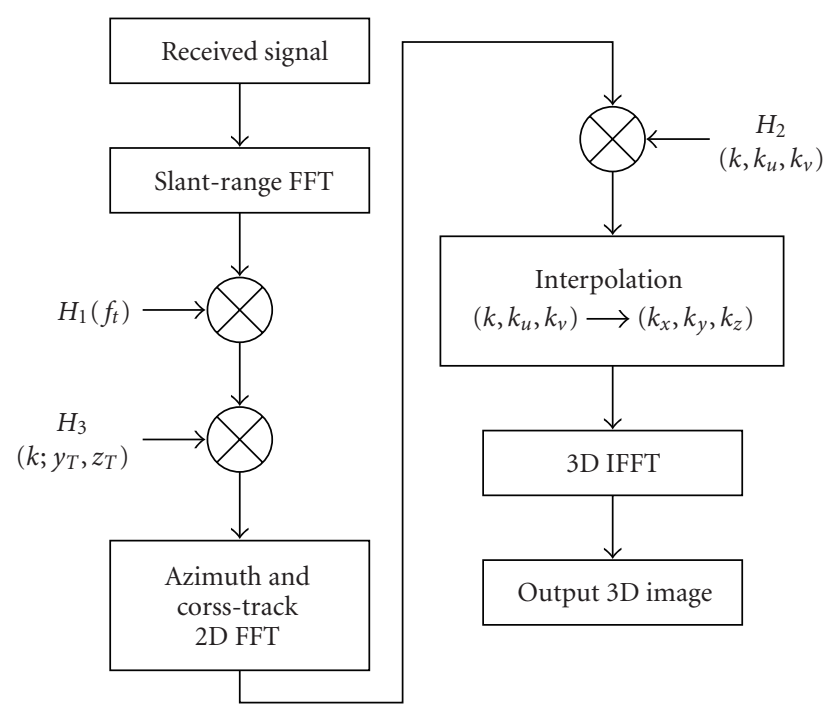

FIGURE 2: Block scheme of 3D RMA for 3D-SAR with STMR-LAA.

(a) Fourier transform along the slant-range direction is firstly applied to the received signal, and the slantrange compression is performed with the frequency domain matched filter $H_{1}\left(f_{t}\right)$.

(b) The nonlinear phase terms are compensated by multiplying the wavenumber domain filter $H_{3}\left(k ; y_{T}, z_{T}\right)$, where some approximations are adopted in this step to improve the computational efficiency.

(c) After the 2D Fourier transform along the azimuth and cross-track directions, constant phase term is removed by multiplying $H_{2}\left(k, k_{u}, k_{v}\right)$ in $3 \mathrm{D}$ wavenumber domain.

(d) A 3D wavenumber mapping is proposed to transform the signal in the $\left(k, k_{u}, k_{v}\right)$ domain into the one in the $\left(k_{x}, k_{y}, k_{z}\right)$ domain, and an interpolation is done to make the signal evenly sampled in the $\left(k_{x}, k_{y}, k_{z}\right)$ domain. The interpolation can be separated into two steps, where the first step is $k_{3}=k+\sqrt{k^{2}-k_{v}^{2}}$ and the following one is $k_{z}=-\sqrt{k_{3}^{2}-k_{u}^{2}}$.

(e) 3D inverse Fourier transform is performed to generate the final 3D image in the spatial domain OXYZ.

\section{Simulation Results}

For evaluating the proposed algorithm, we carry out some simulations for 3D-SAR with STMR-LAA in the downwardlooking mode. Simulation parameters are given in Table 1. The values of the platform parameters correspond to the characteristics of a small UAV.

Consider a $50 \mathrm{~m} \times 50 \mathrm{~m} \times 50 \mathrm{~m}$ (azimuth $\times$ ground range $\times$ elevation) target region composed of 28 point targets that are located at the nadir area under the platform in $3 \mathrm{D}$ spatial domain OXYZ. The distribution of point targets is shown in Figure 3. 


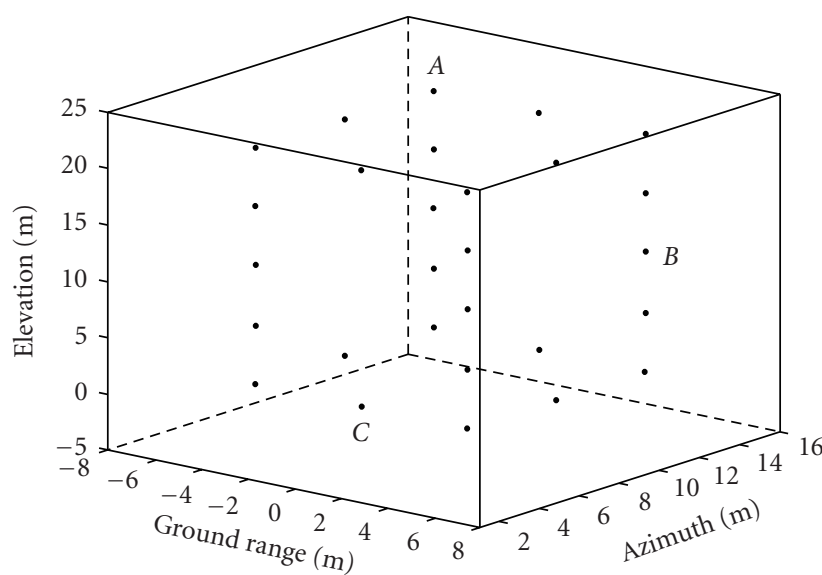

(a)

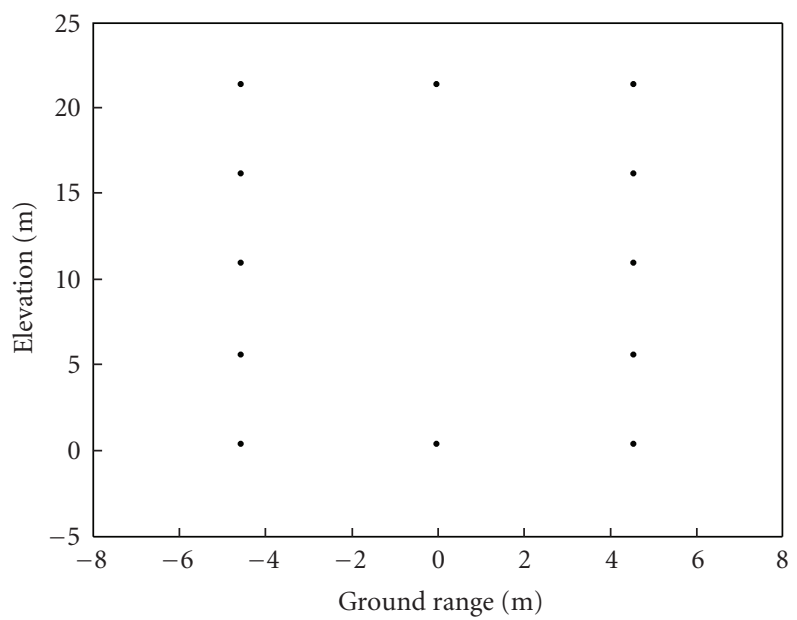

(c)

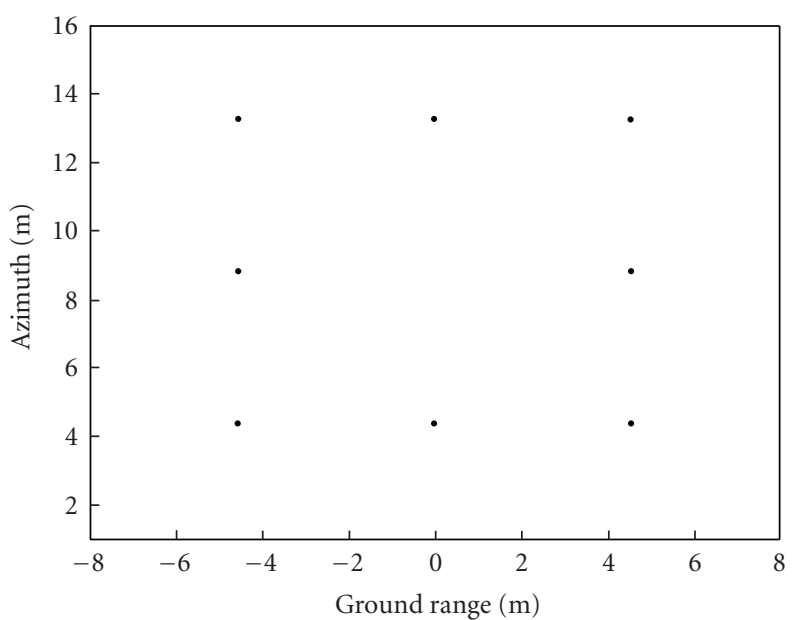

(b)

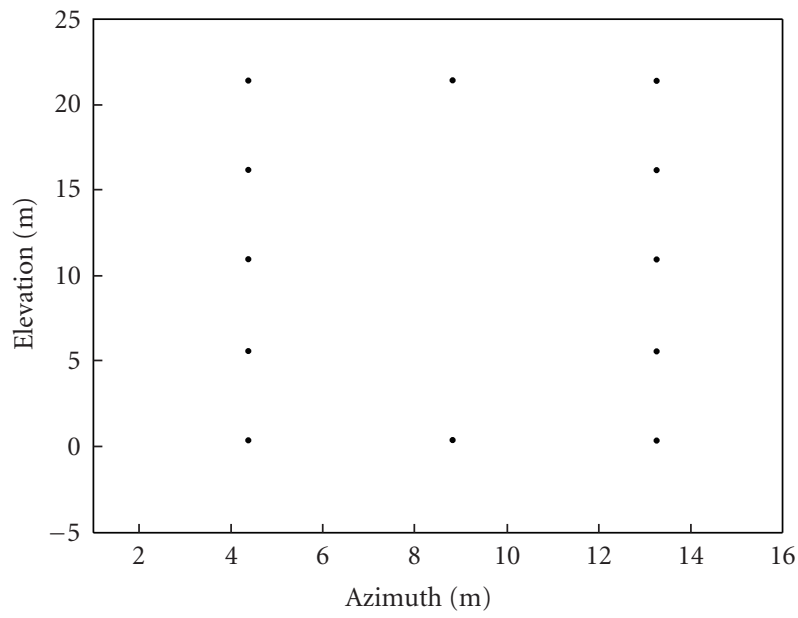

(d)

FIgURE 3: Point targets distribution. (a) the point targets in the 3D spatial domain OXYZ; (b) projections onto the $2 \mathrm{D}$ OXY plane; (c) projections onto the $2 \mathrm{D} O Y Z$ plane; (d) projections onto the $2 \mathrm{D} O X Z$ plane.

According to (46) and simulation parameters in Table 1, we can obtain that $q_{\max }=1.9 \times 10^{-5}$. Figure 4 shows the nonlinear phase terms shown in (47) and the nonlinear phase error between $H_{3}\left(k, k_{u}, k_{v} ; y_{T}, z_{T}\right)$ in $(41)$ and $H_{3}\left(k ; y_{T}, z_{T}\right)$ in (47), where $y_{T}$ and $z_{T}$ are chosen at the borders of the illuminated scenario. It can be seen that nonlinear phase errors are all smaller than $\pi / 4$ radian at the three wavenumber points $k_{\min }, k_{c}$, and $k_{\max }$ and can be negligible compared with the corresponding nonlinear phase terms. Therefore, it will not deteriorate the focusing quality by the use of the approximate expression (47) to compensate the nonlinear phase terms.

With the 3D RMA proposed in this paper, the 3D imaging results of the 28 point targets are shown in Figure 5, where an oversampling factor of 8 has been used in 5(b)5(d) to enhance the intensity of the image display and no windowing is used in the processing. The $3 \mathrm{D}$ image is displayed at the threshold of $-3 \mathrm{~dB}$ as shown in Figure 5(a), and the positions of the 28 point targets are consistent with the real situations in Figure 3(a). Figures 5(b) and 5(c) show
TABLE 1: Simulation parameters.

\begin{tabular}{lc}
\hline Altitude $(H)$ & $1 \mathrm{~km}$ \\
Velocity $\left(v_{0}\right)$ & $40 \mathrm{~m} / \mathrm{s}$ \\
Carrier frequency $\left(f_{c}\right)$ & $37.5 \mathrm{GHz}$ \\
Bandwidth $(B)$ & $300 \mathrm{MHz}$ \\
Pulse repetition frequency & $200 \mathrm{~Hz}$ \\
Length of LAA $(L)$ & $16 \mathrm{~m}$ \\
Number of Rx $(N)$ & 256 \\
Azimuth beamwidth of Tx and & \\
Rx $\left(\Theta_{T}\right.$ and $\left.\Theta_{R}\right)$ & $0.5^{\circ}$ \\
Cross-track beamwidth of Rx & \\
$\left(\Phi_{R}\right)$ & $3^{\circ}$ \\
Theoretical resolution (azimuth & \\
$\times$ ground - range $\times$ elevation) & $0.5 \mathrm{~m} \times 0.5 \mathrm{~m} \times 0.5 \mathrm{~m}$ \\
\hline
\end{tabular}

the $2 \mathrm{D}$ image at different sections, which agree with the theoretical values shown in Figures 3(b) and 3(c). 


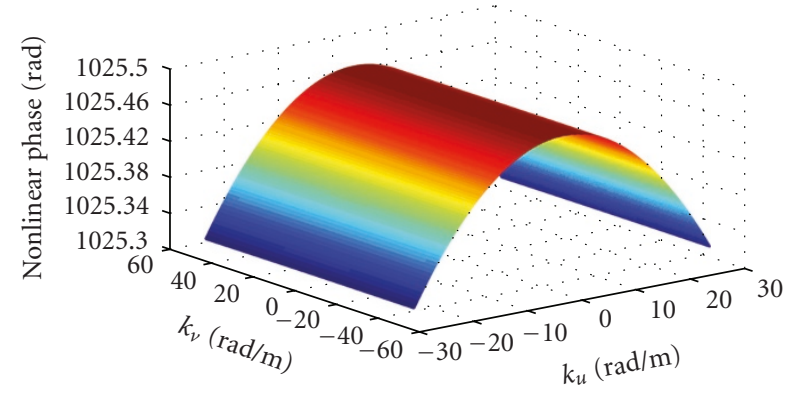

(a)

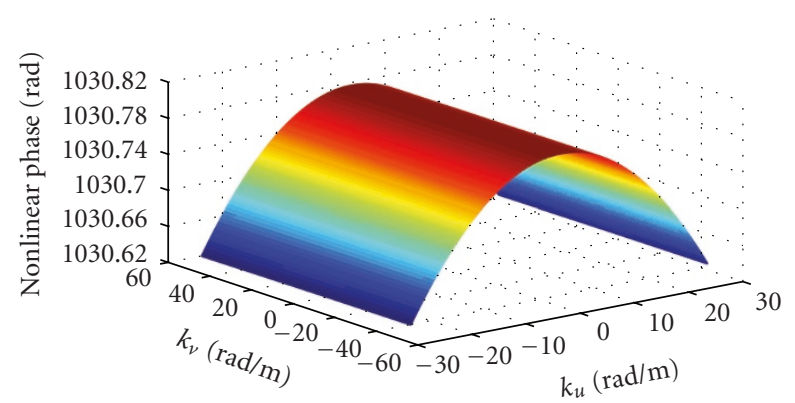

(c)

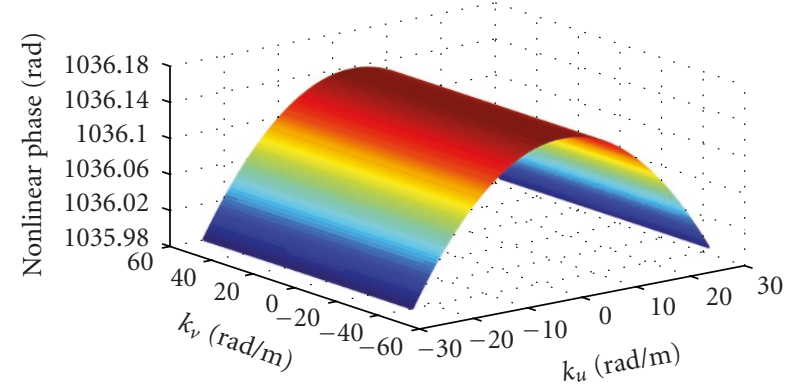

(e)

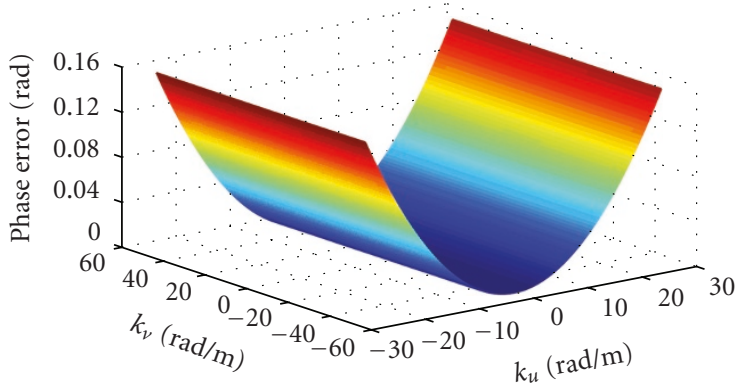

(b)

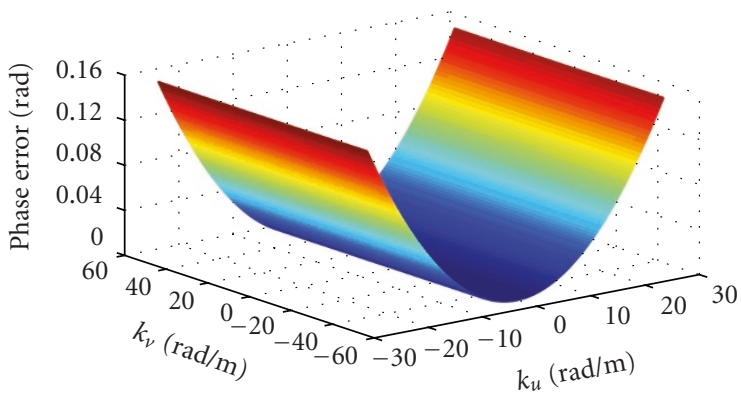

(d)

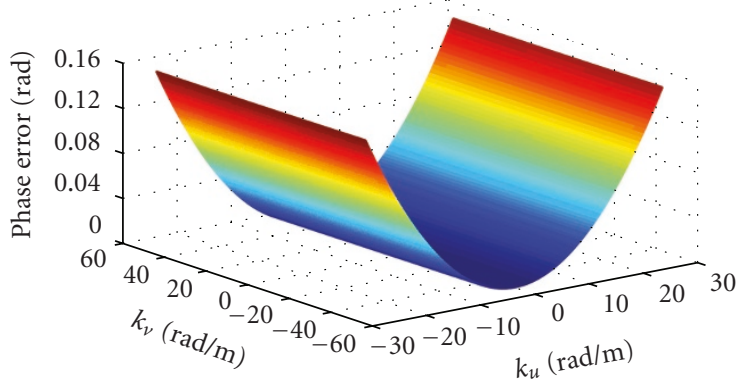

(f)

FIGURE 4: The nonlinear phase terms shown in (41) and nonlinear phase error between (41) and (47). (a) nonlinear phase terms calculated at $k=k_{\min }$; (b) nonlinear phase error calculated at $k=k_{\min }$; (c) nonlinear phase terms calculated at $k=k_{c}$; (d) nonlinear phase error calculated at $k=k_{c}$; (e) nonlinear phase terms calculated at $k=k_{\max }$; (f) nonlinear phase error calculated at $k=k_{\max }$.

TABLE 2: Image quality parameters.

\begin{tabular}{lcccccccr}
\hline Point target & \multicolumn{3}{c}{ Resolution $(\mathrm{m})$} & \multicolumn{3}{c}{ PSLR $(\mathrm{dB})$} & \multicolumn{3}{c}{ ISLR (dB) } \\
& $X$ & $Y$ & $Z$ & $X$ & $Y$ & $Z$ & $X$ & $Y$ \\
\hline A & 0.51 & 0.52 & 0.53 & -13.23 & -13.15 & -13.18 & -10.40 & -10.34 \\
B & 0.52 & 0.51 & 0.53 & -13.20 & -13.17 & -13.21 & -10.41 & -10.20 \\
C & 0.51 & 0.51 & 0.52 & -13.24 & -13.23 & -13.26 & -10.25 & -10.35 \\
\hline
\end{tabular}

Three point targets A, B and C shown in Figure 3(a) are chosen to verify the performance and focusing quality of the proposed 3D RMA, and the image quality parameters, including the resolution, the peak sidelobe ratio (PSLR) and the integrated sidelobe ratio (ISLR), are shown in Table 2. From the above simulation results, we can see that the point targets are all well focused after the removal of constant phase term and compensation of nonlinear phase terms, and the expansion of $\Phi_{0}$ in (20) is effective without taking the quartic and higher-order terms into account.

\section{Influence of Platform Deviations}

During the analysis mentioned above, the platform which carries the 3D-SAR system is assumed to fly along a straight line with the constant velocity at the invariable altitude. Our 


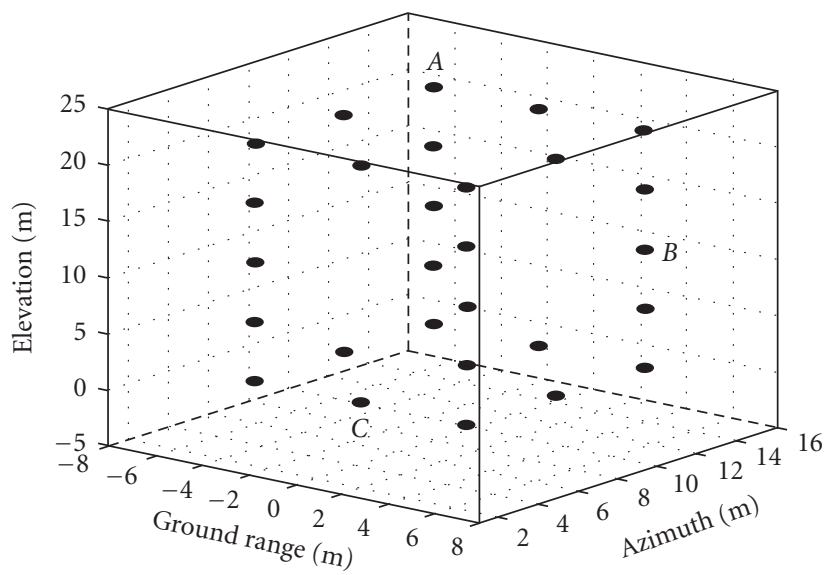

(a)

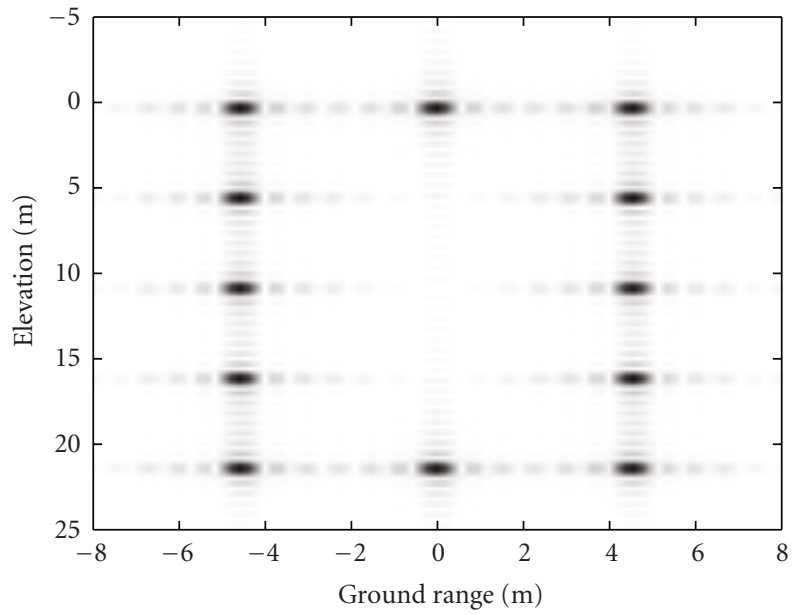

(c)

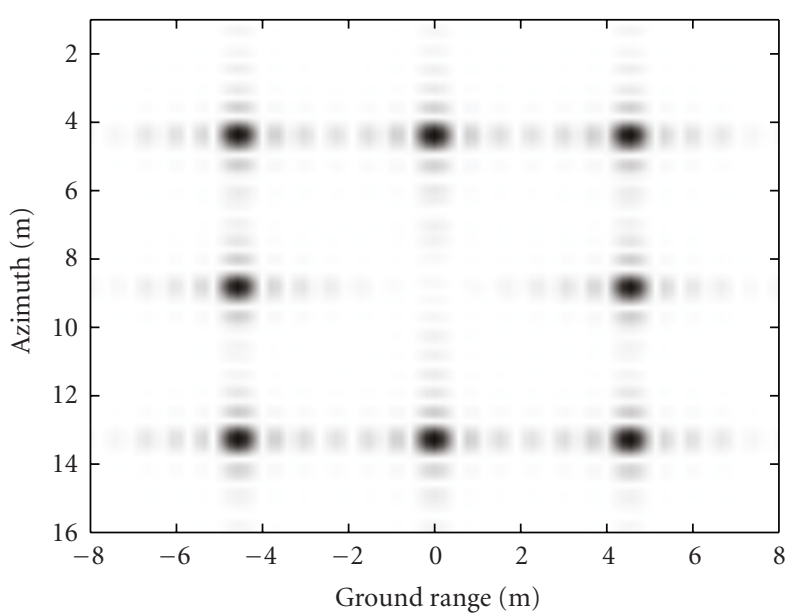

(b)

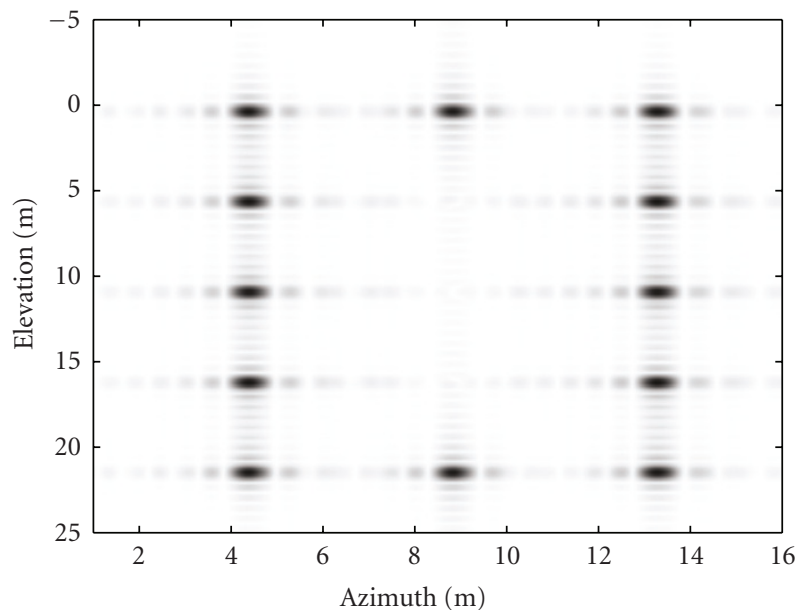

(d)

Figure 5: The imaging results. (a) the $3 \mathrm{D}$ imaging results, where the surfaces of the data are plotted at $-3 \mathrm{~dB}$; (b) the $2 \mathrm{D}$ imaging results on the $2 \mathrm{D} O X Y$ plane, where $Z=0.5 \mathrm{~m}$; (c) the $2 \mathrm{D}$ imaging results on the $2 \mathrm{D} O Y Z$ plane, where $X=4.5 \mathrm{~m}$; (d) the $2 \mathrm{D}$ imaging results on the 2D OXZ plane, where $Y=4.5 \mathrm{~m}$.

present 3D-SAR with STMR-LAA system is based on the ASTRO, which is a ground-based SAR platform developed at MITL-IECAS. It works in the anechoic chamber and on the ground, and positioning precision as well as control accuracy is high enough to satisfy the above assumption. However, when the system is mounted on the airplane or helicopter platform, trajectory deviations from the straight line, attitude disturbances and forward velocity variations are unavoidable due to atmospheric turbulence. Moreover, the wing oscillation during flying continuously changes the position of every antenna element. These will introduce motion errors to the received raw signal, and may severely impair the final 3D image quality. The impact of platform attitude disturbances on the 3D image quality is presented in Reference [14], and the 3D image quality analysis of the displacement of antenna elements caused by the wing oscillation is given in [12]. In this paper, we analyze the impact of platform trajectory deviations on the 3D image quality, and present the required accuracy on the platform position to generate the $3 \mathrm{D}$ images.

The imaging geometry in the presence of trajectory deviations is shown in Figure 6, where the system axes coincide with the ideal trajectory case shown in Figure 1. The deviation vector $\mathbf{b}$ equals to $b_{x} \mathbf{i}+b_{y} \mathbf{j}+b_{z} \mathbf{k}$ in the Cartesian coordinates, where $b_{x}, b_{y}$ and $b_{z}$ are the deviation components in the $x$-axis, $y$-axis and $z$-axis, respectively. An analysis on the effect of more complex motion deviation trajectories (pitch-, yaw-, and roll-angle variations) is out of the scope of the paper. The deviation component in the $x$-axis is caused by the variable velocity which results in unevenly spaced samples in the azimuth direction, and can be compensated via a proper resampling of the received raw signal. Thus it is assumed in the following analysis that the platform trajectory deviation in the $x$-axis has been compensated and $b_{x}$ is null. Then the simplified imaging geometry on the zero Doppler plane is shown in Figure 7, 


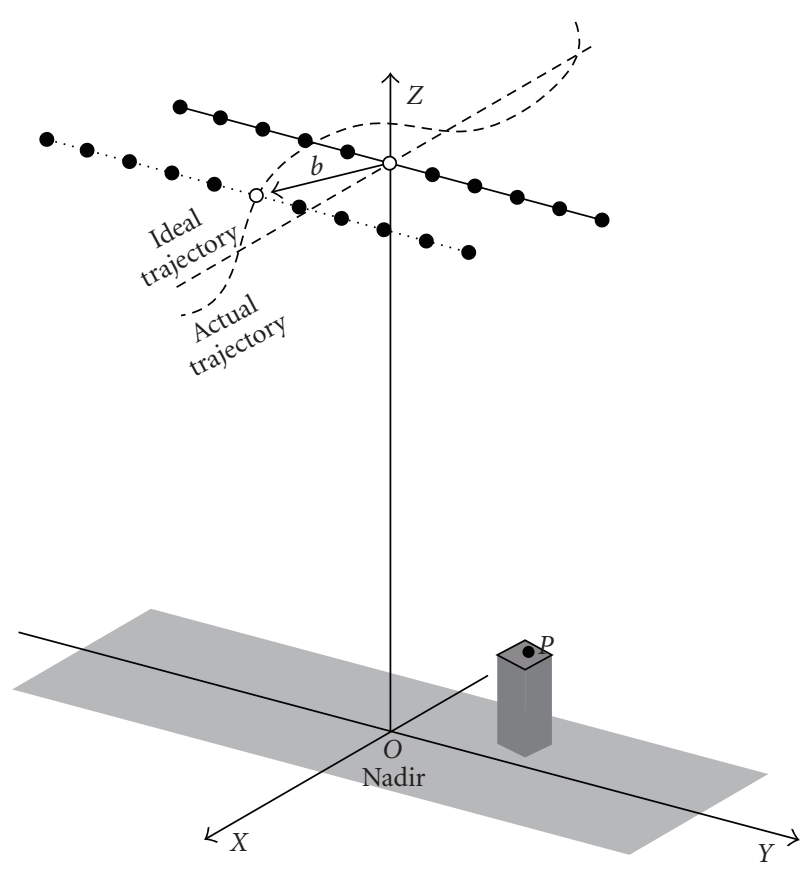

FIGURE 6: Geometry in presence of trajectory deviations.

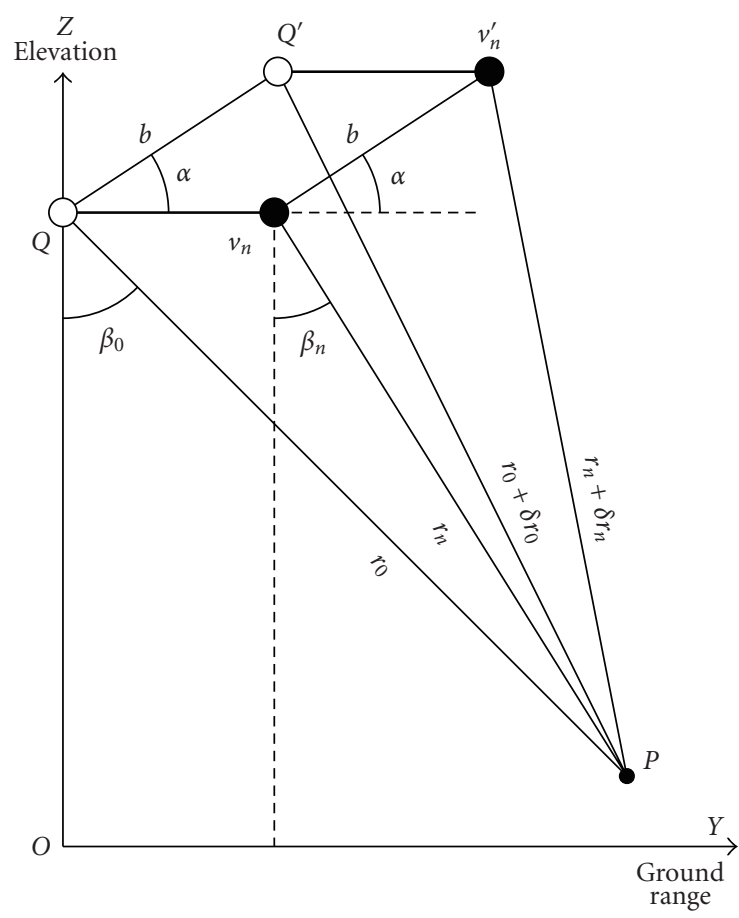

FIgURE 7: Geometry on zero Doppler plane. where $r_{0}$ is the distance from the Tx to the target $P$ in the case of ideal trajectory; $r_{0}+\delta r_{0}$ is the distance from the Tx to the target $P$ in the case of actual trajectory; $r_{n}$ is the distance from the target $P$ to the $n$th $\mathrm{Rx}$ in the case of ideal trajectory; $r_{n}+\delta r_{n}$ is the distance from the target $P$ to the $n$th $\mathrm{Rx}$ in the case of actual trajectory; $\beta_{0}$ denotes the look angle from the Tx to the target $P ; \beta_{n}$ denotes the look angle from the $n$th $\mathrm{Rx}$ to the target $P$. The deviation vector $\mathbf{b}$ equals to $b(\cos \alpha \mathbf{j}+\sin \alpha \mathbf{k})$ in the polar coordinates.

According to the Cosine Theorem [21], we have

$$
r_{0}+\delta r_{0}=\sqrt{r_{0}^{2}+b^{2}-2 r_{0} b \sin \left(\beta_{0}-\alpha\right)} .
$$

When the value of the deviation is small compared with the slant range of the target, the range error $\delta r_{0}$ in (51) can be approximately expressed as $\delta r_{0} \approx b \sin \left(\alpha-\beta_{0}\right)$. Likewise, the range error $\delta r_{n}$ can be calculated as $\delta r_{n} \approx b \sin (\alpha-$ $\left.\beta_{n}\right)$. According to (16), the phase error caused by trajectory deviations can be expressed as

$$
\delta \Phi=k\left(\delta r_{0}+\delta r_{n}\right) \approx 2 k_{c} b \sin \left(\alpha-\frac{\beta_{0}+\beta_{n}}{2}\right) \cos \left(\frac{\beta_{0}-\beta_{n}}{2}\right) .
$$

The phase error $\delta \Phi$ varies with the polar angle $\alpha$ when the polar radius $b$ is constant. The absolute maximum of $\delta \Phi$ is obtained at $\alpha= \pm \pi / 2+\left(\beta_{0}+\beta_{n}\right) / 2$, and the absolute minimum, which equals to zero, is obtained at $\alpha=\left(\beta_{0}+\beta_{n}\right) / 2$ and $\alpha=\pi+\left(\beta_{0}+\beta_{n}\right) / 2$. Therefore, the whole trajectory deviations can be separate into the deviation component along the equivalent target look direction (i.e., $\alpha= \pm \pi / 2+$ $\left.\left(\beta_{0}+\beta_{n}\right) / 2\right)$ and the deviation component perpendicular to the equivalent target look direction (i.e., $\alpha=\left(\beta_{0}+\right.$ $\left.\beta_{n}\right) / 2$ and $\left.\alpha=\pi+\left(\beta_{0}+\beta_{n}\right) / 2\right)$. The second deviation component contributes nothing to the phase error, and the compensation of platform trajectory deviations only needs to take the first component into account.

The impact of platform trajectory deviations on the 3D image quality can be ignored when the magnitude of phase error caused by trajectory deviations is smaller than $\pi / 4$ [22]. According to this principle, the range of required accuracy on the platform position should satisfy the following inequation to generate the 3D image:

$$
\left|b_{z} \cos \left(\frac{\beta_{0}+\beta_{n}}{2}\right)-b_{y} \sin \left(\frac{\beta_{0}+\beta_{n}}{2}\right)\right| \leq \frac{\lambda_{c}}{16 \cos \left[\left(\beta_{0}-\beta_{n}\right) / 2\right]},
$$

where $b_{y}=b \cos \alpha$ and $b_{z}=b \sin \alpha$. It can be calculated with the parameters given in Table 1 that the platform position should be known with accuracy up to the magnitude of millimeter. For platform deviations larger than that, the PSLR and ISLR will be increased drastically. It demonstrates that the platform position has to be measured or estimated very precisely in order to form high quality $3 \mathrm{D}$ images.

\section{Conclusions}

This paper presents a 3D RMA suitable for downwardlooking 3D-SAR with STMR-LAA. The precise implicit expression of the signal spectrum in $3 \mathrm{D}$ wavenumber domain is firstly described. Then the approximate explicit expression of the signal spectrum is derived by expanding the implicit phase term to its Taylor series. After the removal of constant 
phase term and compensation of nonlinear phase terms, a $3 \mathrm{D}$ wavenumber mapping is proposed to replace the wavenumber in the direction of wave propagation by the wavenumber observed in $3 \mathrm{D}$ spatial domain $O X Y Z$, and an interpolation is performed to transform these unevenly spaced samples into evenly spaced ones in $3 \mathrm{D}$ wavenumber domain. The simulation results about the imaging quality and the phase error analysis validate the feasibility of the imaging algorithm. Furthermore, the impact of platform trajectory deviations on the 3D image quality is discussed, and it concludes that the platform position has to be known precisely to form high quality $3 \mathrm{D}$ images.

Future work will extend the proposed 3D RMA to more complex bistatic configurations of downward-looking 3D-SAR with LAA, such as multi-transmitting and multireceiving case. In addition, this paper contains neither technological aspects about the realization of airborne 3DSAR with LAA system nor the key techniques, including the motion compensation, wings oscillation compensation and relevant problems. These special problems have to be treated separately when the 3D-SAR with LAA system will be mounted on the airplane or helicopter platform in the future.

\section{Appendices}

\section{A. Derivation of the First Partial Derivatives of $\Phi_{0}$ with Respect To $y_{T}$ and $z_{T}$}

According to (16), the first partial derivative of $\Phi_{0}$ with respect to $y_{T}$ can be written as

$$
\frac{\partial \Phi_{0}}{\partial y_{T}}=k \frac{\partial R\left(U_{0}, V_{0}\right)}{\partial y_{T}}+k_{u} \frac{\partial U_{0}}{\partial y_{T}}+k_{v} \frac{\partial V_{0}}{\partial y_{T}},
$$

where

$$
\begin{gathered}
R\left(U_{0}, V_{0}\right)=R_{T}\left(U_{0}\right)+R_{R}\left(U_{0}, V_{0}\right), \\
R_{T}\left(U_{0}\right)=\sqrt{U_{0}^{2}+y_{T}^{2}+\left(H-z_{T}\right)^{2}}, \\
R_{R}\left(U_{0}, V_{0}\right)=\sqrt{U_{0}^{2}+V_{0}^{2}+\left(H-z_{T}\right)^{2}} .
\end{gathered}
$$

We can see from (A.2) that $R\left(U_{0}, V_{0}\right)$ are the function of $U_{0}$, $V_{0}, y_{T}$ and $z_{T}$, and the stationary phase points $U_{0}$ and $V_{0}$ are the function of $y_{T}$ and $z_{T}$. Therefore,

$$
\begin{aligned}
\frac{\partial R\left(U_{0}, V_{0}\right)}{\partial y_{T}}= & R_{y_{T}}\left(U_{0}, V_{0}\right)+R_{U_{0}}\left(U_{0}, V_{0}\right) \frac{\partial U_{0}}{\partial y_{T}} \\
& +R_{V_{0}}\left(U_{0}, V_{0}\right) \frac{\partial V_{0}}{\partial y_{T}} \\
= & \frac{y_{T}}{R_{T}\left(U_{0}\right)}+\left[\frac{U_{0}}{R_{T}\left(U_{0}\right)}+\frac{U_{0}}{R_{R}\left(U_{0}, V_{0}\right)}\right] \frac{\partial U_{0}}{\partial y_{T}} \\
& +\frac{V_{0}}{R_{R}\left(U_{0}, V_{0}\right)} \frac{\partial V_{0}}{\partial y_{T}},
\end{aligned}
$$

where $R_{y_{T}}\left(U_{0}, V_{0}\right), R_{U_{0}}\left(U_{0}, V_{0}\right)$ and $R_{V_{0}}\left(U_{0}, V_{0}\right)$ denote the first derivatives of $R\left(U_{0}, V_{0}\right)$ with respect to $y_{T}, U_{0}$ and $V_{0}$. Inserting (A.5) into (A.1) yields

$$
\begin{aligned}
\frac{\partial \Phi_{0}}{\partial y_{T}}= & k \frac{y_{T}}{\sqrt{U_{0}^{2}+y_{T}^{2}+\left(H-z_{T}\right)^{2}}} \\
+ & {\left[k \frac{V_{0}}{\sqrt{U_{0}^{2}+V_{0}^{2}+\left(H-z_{T}\right)^{2}}}+k_{v}\right] \frac{\partial V_{0}}{\partial y_{T}} } \\
+ & {\left[k \frac{U_{0}}{\sqrt{U_{0}^{2}+y_{T}^{2}+\left(H-z_{T}\right)^{2}}}\right.} \\
& \left.+k \frac{U_{0}}{\sqrt{U_{0}^{2}+V_{0}^{2}+\left(H-z_{T}\right)^{2}}}+k_{u}\right] \frac{\partial U_{0}}{\partial y_{T}} .
\end{aligned}
$$

According to equations set (14), the terms in the two square brackets of (A.6) are equal to zero. Therefore, (A.6) can be simplified to be

$$
\frac{\partial \Phi_{0}}{\partial y_{T}}=k \frac{y_{T}}{\sqrt{U_{0}^{2}+y_{T}^{2}+\left(H-z_{T}\right)^{2}}} .
$$

Likewise, the first partial derivative of $\Phi_{0}$ with respect to $z_{T}$ can be calculated.

$$
\begin{aligned}
& \frac{\partial \Phi_{0}}{\partial z_{T}} \\
& =k \frac{\partial R\left(U_{0}, V_{0}\right)}{\partial z_{T}}+k_{u} \frac{\partial U_{0}}{\partial z_{T}}+k_{v} \frac{\partial V_{0}}{\partial z_{T}} \\
& =k\left\{\frac{z_{T}-H}{\sqrt{U_{0}^{2}+y_{T}^{2}+\left(H-z_{T}\right)^{2}}}+\frac{z_{T}-H}{\sqrt{U_{0}^{2}+V_{0}^{2}+\left(H-z_{T}\right)^{2}}}\right\} \\
& +\left[k \frac{V_{0}}{\sqrt{U_{0}^{2}+V_{0}^{2}+\left(H-z_{T}\right)^{2}}}+k_{v}\right] \frac{\partial V_{0}}{\partial z_{T}} \\
& +\left[k \frac{U_{0}}{\sqrt{U_{0}^{2}+y_{T}^{2}+\left(H-z_{T}\right)^{2}}}\right. \\
& \left.+k \frac{U_{0}}{\sqrt{U_{0}^{2}+V_{0}^{2}+\left(H-z_{T}\right)^{2}}}+k_{u}\right] \frac{\partial U_{0}}{\partial z_{T}} \\
& =\frac{z_{T}-H}{U_{0}}\left\{k \frac{U_{0}}{\sqrt{U_{0}^{2}+y_{T}^{2}+\left(H-z_{T}\right)^{2}}}\right. \\
& \left.+k \frac{U_{0}}{\sqrt{U_{0}^{2}+V_{0}^{2}+\left(H-z_{T}\right)^{2}}}\right\} \\
& =k_{u} \frac{H-z_{T}}{U_{0}} .
\end{aligned}
$$




\section{B. Derivation of the First Partial Derivatives of $U_{0}$ and $V_{0}$ with Respect to $y_{T}$ and $z_{T}$ \\ Evaluated at $y_{T}=0, z_{T}=0$}

The first partial derivatives of equations set (14) with respect to $y_{T}$ can be written as

$$
\begin{gathered}
k \frac{\partial}{\partial y_{T}}\left[\frac{U_{0}}{\sqrt{U_{0}^{2}+y_{T}^{2}+\left(H-z_{T}\right)^{2}}}+\frac{U_{0}}{\sqrt{U_{0}^{2}+V_{0}^{2}+\left(H-z_{T}\right)^{2}}}\right]=0, \\
k \frac{\partial}{\partial y_{T}}\left[\frac{V_{0}}{\sqrt{U_{0}^{2}+V_{0}^{2}+\left(H-z_{T}\right)^{2}}}\right]=0 .
\end{gathered}
$$

Expanding (B.1), we have

$$
\begin{gathered}
-\frac{y_{T} U_{0}}{R_{T}^{3}\left(U_{0}\right)}+\left[\frac{y_{T}^{2}+\left(H-z_{T}\right)^{2}}{R_{T}^{3}\left(U_{0}\right)}+\frac{V_{0}^{2}+\left(H-z_{T}\right)^{2}}{R_{R}^{3}\left(U_{0}, V_{0}\right)}\right] \frac{\partial U_{0}}{\partial y_{T}} \\
-\frac{U_{0} V_{0}}{R_{R}^{3}\left(U_{0}, V_{0}\right)} \frac{\partial V_{0}}{\partial y_{T}}=0, \\
-\frac{U_{0} V_{0}}{R_{R}^{3}\left(U_{0}, V_{0}\right)} \frac{\partial U_{0}}{\partial y_{T}}+\frac{U_{0}^{2}+\left(H-z_{T}\right)^{2}}{R_{R}^{3}\left(U_{0}, V_{0}\right)} \frac{\partial V_{0}}{\partial y_{T}}=0 .
\end{gathered}
$$

Therefore, the first partial derivates of $U_{0}$ and $V_{0}$ with respect to $y_{T}$ evaluated at $y_{T}=0, z_{T}=0$ can be calculated as follows:

$$
\begin{gathered}
{\left.\left[\frac{H^{2}}{\left(U_{00}^{2}+H^{2}\right)^{3 / 2}}+\frac{V_{00}^{2}+H^{2}}{\left(U_{00}^{2}+V_{00}^{2}+H^{2}\right)^{3 / 2}}\right] \frac{\partial U_{0}}{\partial y_{T}}\right|_{y_{T}=0, z_{T}=0}} \\
-\left.\frac{U_{00} V_{00}}{\left(U_{00}^{2}+V_{00}^{2}+H^{2}\right)^{3 / 2}} \frac{\partial V_{0}}{\partial y_{T}}\right|_{y_{T}=0, z_{T}=0}=0, \\
-\left.U_{00} V_{00} \frac{\partial U_{0}}{\partial y_{T}}\right|_{y_{T}=0, z_{T}=0}=0 . \\
+\left.\left(U_{00}^{2}+H^{2}\right) \frac{\partial V_{0}}{\partial y_{T}}\right|_{y_{T}=0, z_{T}=0}=0 .
\end{gathered}
$$

For the determinant of coefficient matrix in equations set (B.3) is unequal to zero, that is,

$$
\begin{gathered}
\left|\begin{array}{cc}
\frac{H^{2}}{\left(U_{00}^{2}+H^{2}\right)^{3 / 2}}+\frac{V_{00}^{2}+H^{2}}{\left(U_{00}^{2}+V_{00}^{2}+H^{2}\right)^{3 / 2}} & \frac{-U_{00} V_{00}}{\left(U_{00}^{2}+V_{00}^{2}+H^{2}\right)^{3 / 2}} \\
-U_{00} V_{00} & U_{00}^{2}+H^{2}
\end{array}\right| \\
=\frac{H^{2}}{\sqrt{U_{00}^{2}+H^{2}}}+\frac{H^{2}}{\sqrt{U_{00}^{2}+V_{00}^{2}+H^{2}}} \neq 0,
\end{gathered}
$$

the solution of equations set (B.3) is

$$
\begin{aligned}
& \left.\frac{\partial U_{0}}{\partial y_{T}}\right|_{y_{T}=0, z_{T}=0}=0, \\
& \left.\frac{\partial V_{0}}{\partial y_{T}}\right|_{y_{T}=0, z_{T}=0}=0 .
\end{aligned}
$$

Likewise, the first partial derivatives of equations set (14) with respect to $z_{T}$ can be written as

$$
\begin{gathered}
k \frac{\partial}{\partial z_{T}}\left[\frac{U_{0}}{\sqrt{U_{0}^{2}+y_{T}^{2}+\left(H-z_{T}\right)^{2}}}+\frac{U_{0}}{\sqrt{U_{0}^{2}+V_{0}^{2}+\left(H-z_{T}\right)^{2}}}\right]=0, \\
k \frac{\partial}{\partial z_{T}}\left[\frac{V_{0}}{\sqrt{U_{0}^{2}+V_{0}^{2}+\left(H-z_{T}\right)^{2}}}\right]=0 .
\end{gathered}
$$

Expanding (B.6), we have

$$
\begin{aligned}
& \frac{U_{0}\left(H-z_{T}\right)}{R_{T}^{3}\left(U_{0}\right)}+\frac{U_{0}\left(H-z_{T}\right)}{R_{R}^{3}\left(U_{0}, V_{0}\right)} \\
& \quad+\left[\frac{y_{T}^{2}+\left(H-z_{T}\right)^{2}}{R_{T}^{3}\left(U_{0}\right)}+\frac{V_{0}^{2}+\left(H-z_{T}\right)^{2}}{R_{R}^{3}\left(U_{0}, V_{0}\right)}\right] \frac{\partial U_{0}}{\partial z_{T}} \\
& \quad-\frac{U_{0} V_{0}}{R_{R}^{3}\left(U_{0}, V_{0}\right)} \frac{\partial V_{0}}{\partial z_{T}}=0, \\
& \frac{V_{0}\left(H-z_{T}\right)}{R_{R}^{3}\left(U_{0}, V_{0}\right)}-\frac{U_{0} V_{0}}{R_{R}^{3}\left(U_{0}, V_{0}\right)} \frac{\partial U_{0}}{\partial z_{T}} \\
& \quad+\frac{U_{0}^{2}+\left(H-z_{T}\right)^{2}}{R_{R}^{3}\left(U_{0}, V_{0}\right)} \frac{\partial V_{0}}{\partial z_{T}}=0 .
\end{aligned}
$$

Therefore, the first partial derivates of $U_{0}$ and $V_{0}$ with respect to $z_{T}$ evaluated at $y_{T}=0, z_{T}=0$ can be calculated as follows:

$$
\begin{aligned}
& \frac{U_{00} H}{\left(U_{00}^{2}+H^{2}\right)^{3 / 2}}+\frac{U_{00} H}{\left(U_{00}^{2}+V_{00}^{2}+H^{2}\right)^{3 / 2}} \\
& +\left.\left[\frac{H^{2}}{\left(U_{00}^{2}+H^{2}\right)^{3 / 2}}+\frac{V_{00}^{2}+H^{2}}{\left(U_{00}^{2}+V_{00}^{2}+H^{2}\right)^{3 / 2}}\right] \frac{\partial U_{0}}{\partial z_{T}}\right|_{y_{T}=0, z_{T}=0} \\
& -\left.\frac{U_{00} V_{00}}{\left(U_{00}^{2}+V_{00}^{2}+H^{2}\right)^{3 / 2}} \frac{\partial V_{0}}{\partial z_{T}}\right|_{y_{T}=0, z_{T}=0}=0, \\
& V_{00} H-\left.U_{00} V_{00} \frac{\partial U_{0}}{\partial z_{T}}\right|_{y_{T}=0, z_{T}=0}=0 . \\
& +\left.\left(U_{00}^{2}+H^{2}\right) \frac{\partial V_{0}}{\partial z_{T}}\right|_{y_{T}=0, z_{T}=0}=0
\end{aligned}
$$

Solving the equations set (B.8), we obtain

$$
\begin{aligned}
& \left.\frac{\partial U_{0}}{\partial z_{T}}\right|_{y_{T}=0, z_{T}=0}=-\frac{U_{00}}{H}, \\
& \left.\frac{\partial V_{0}}{\partial z_{T}}\right|_{y_{T}=0, z_{T}=0}=-\frac{V_{00}}{H} .
\end{aligned}
$$




\section{Derivation of the Second Partial Derivatives of $U_{0}$ and $V_{0}$ with Respect to $z_{T}$ Evaluated at $y_{T}=0, z_{T}=0$}

The first partial derivatives of equations set (B.7) with respect to $z_{T}$ can be written as

$$
\begin{aligned}
& \frac{\partial}{\partial z_{T}}\left\{\frac{U_{0}\left(H-z_{T}\right)}{R_{T}^{3}\left(U_{0}\right)}+\frac{U_{0}\left(H-z_{T}\right)}{R_{R}^{3}\left(U_{0}, V_{0}\right)}\right. \\
& +\left[\frac{y_{T}^{2}+\left(H-z_{T}\right)^{2}}{R_{T}^{3}\left(U_{0}\right)}+\frac{V_{0}^{2}+\left(H-z_{T}\right)^{2}}{R_{R}^{3}\left(U_{0}, V_{0}\right)}\right] \frac{\partial U_{0}}{\partial z_{T}} \\
& \left.\quad-\frac{U_{0} V_{0}}{R_{R}^{3}\left(U_{0}, V_{0}\right)} \frac{\partial V_{0}}{\partial z_{T}}\right\}=0, \\
& \frac{\partial}{\partial z_{T}}\left\{\frac{V_{0}\left(H-z_{T}\right)}{R_{R}^{3}\left(U_{0}, V_{0}\right)}-\frac{U_{0} V_{0}}{R_{R}^{3}\left(U_{0}, V_{0}\right)} \frac{\partial U_{0}}{\partial z_{T}}\right. \\
& \left.+\frac{U_{0}^{2}+\left(H-z_{T}\right)^{2}}{R_{R}^{3}\left(U_{0}, V_{0}\right)} \frac{\partial V_{0}}{\partial z_{T}}\right\}=0 .
\end{aligned}
$$

Expanding (C.1) and inserting (B.9) into it, we can obtain that the second partial derivatives of $U_{0}$ and $V_{0}$ with respect to $z_{T}$ evaluated at $y_{T}=0, z_{T}=0$ satisfy the following equations set, that is,

$$
\begin{gathered}
{\left.\left[\frac{H^{2}}{\left(U_{00}^{2}+H^{2}\right)^{3 / 2}}+\frac{V_{00}^{2}+H^{2}}{\left(U_{00}^{2}+V_{00}^{2}+H^{2}\right)^{3 / 2}}\right] \frac{\partial^{2} U_{0}}{\partial z_{T}^{2}}\right|_{y_{T}=0, z_{T}=0}} \\
-\left.\frac{U_{00} V_{00}}{\left(U_{00}^{2}+V_{00}^{2}+H^{2}\right)^{3 / 2}} \frac{\partial^{2} V_{0}}{\partial z_{T}^{2}}\right|_{y_{T}=0, z_{T}=0}=0 \\
-\left.U_{00} V_{00} \frac{\partial^{2} U_{0}}{\partial z_{T}^{2}}\right|_{y_{T}=0, z_{T}=0}=0 \\
+\left.\left(U_{00}^{2}+H^{2}\right) \frac{\partial^{2} V_{0}}{\partial z_{T}^{2}}\right|_{y_{T}=0, z_{T}=0}=0
\end{gathered}
$$

For equations set (C.2) have the same coefficient matrix with equations set (B.3), we can obtain the solution of equations set (C.2) as follows.

$$
\begin{aligned}
& \left.\frac{\partial^{2} U_{0}}{\partial z_{T}^{2}}\right|_{y_{T}=0, z_{T}=0}=0 \\
& \left.\frac{\partial^{2} V_{0}}{\partial z_{T}^{2}}\right|_{y_{T}=0, z_{T}=0}=0 .
\end{aligned}
$$

\section{Acknowledgments}

This research was supported by Major Program of National Natural Science Foundation of China (Grant no. 60890070), National Natural Science Funds for Distinguished Young Scholar (Grant no. 60725103) and National High Technology Research and Development Program of China (Grant no. 2007AA12Z116). The authors would like to thank Dr.
Zheng-Shu ZHOU of Terrestrial Mapping and Monitoring Stream of CSIRO and Dr. Xiu-Feng SONG of University of Florida for helping improve the English of the manuscript. They also would like to thank the academic editor and anonymous reviewer(s) for their helpful comments which greatly improved the manuscript.

\section{References}

[1] B. R. Mahafza, Two- and three-dimensional synthetic aperture radar imaging using a linear array with transverse motion, Ph.D. dissertation, The University of Alabama in Huntsville, Huntsville, Ala, USA, 1989.

[2] C. H. Gierull, "On a concept for an airborne downwardlooking imaging radar," AEU-Archiv für Elektronik und Ubertragungstechnik, vol. 53, no. 6, pp. 295-304, 1999.

[3] R. Giret, H. Jeuland, and P. Enert, "A study of a 3D-SAR concept for a millimeter-wave imaging radar onboard an UAV," in Proceedings of the 1st European Radar Conference (EURAD '04), pp. 201-204, Amsterdam, The Netherlands, October 2004

[4] J. F. Nouvel, H. Jeuland, G. Bonin, S. Roques, O. du Plessis, and J. Peyret, "A Ka band imaging radar: DRIVE on board ONERA motorglider," in Proceedings of the International Geoscience and Remote Sensing Symposium (IGARSS '06), pp. 134-136, Denver, Colo, USA, August 2006.

[5] J. F. Nouvel, S. Roques, and O. Ruault du Plessis, "A lowcost imaging radar: DRIVE on board ONERA motorglider," in Proceedings of the International Geoscience and Remote Sensing Symposium (IGARSS '07), pp. 5306-5309, Barcelona, Spain, July 2007.

[6] J. F. Nouvel, O. Ruault du Plessis, J. Svedin, and A. Gustafsson, "ONERA DRIVE project," in Proceedings of 7th European Conference on Synthetic Aperture Radar (EUSAR '08), Friedrichshafen, Germany, June 2008.

[7] J. F. Nouvel and O. Ruault du Plessis, "The ONERA compact SAR in Ka band," in Proceedings of 7th European Conference on Synthetic Aperture Radar (EUSAR '08), Friedrichshafen, Germany, June 2008.

[8] J. Klare, A. R. Brenner, and J. H. G. Ender, "A new airborne radar for $3 \mathrm{D}$ imaging-image formation using the ARTINO principle," in Proceedings of 6th European Conference on Synthetic Aperture Radar (EUSAR '06), Dresden, Germany, May 2006.

[9] J. Klare, "A new airborne radar for 3D imaging-simulation study of ARTINO," in Proceedings of 6th European Conference on Synthetic Aperture Radar (EUSAR '06), Dresden, Germany, May 2006.

[10] M. Weiß, J. Ender, O. Peters, and T. Espeter, "An airborne radar for three dimensional imaging and observation-technical realisation and status of ARTINO," in Proceedings of 6th European Conference on Synthetic Aperture Radar (EUSAR '06), Dresden, Germany, May 2006.

[11] J. Klare, M. Weiß, O. Peters, A. R. Brenner, and J. H. G. Ender, "ARTINO: a new high resolution 3D imaging radar system on an autonomous airborne platform," in Proceedings of the International Geoscience and Remote Sensing Symposium (IGARSS '06), Denver, Colo, USA, August 2006.

[12] J. Klare, D. Cerutti-Maori, A. Brenner, and J. Ender, "Image quality analysis of the vibrating sparse MIMO antenna array of the airborne 3D imaging radar ARTINO," in Proceedings of the International Geoscience and Remote Sensing Symposium (IGARSS '07), pp. 5310-5314, Barcelona, Spain, July 2007. 
[13] M. Weiß, O. Peters, and J. Ender, "A three dimensional SAR system on an UAV," in Proceedings of the IEEE International Geoscience and Remote Sensing Symposium (IGARSS '07), pp. 5315-5318, Barcelona, Spain, July 2007.

[14] J. Klare, A. Brenner, and J. Ender, "Impact of platform attitude disturbances on the 3D imaging quality of the UAV ARTINO," in Proceedings of 7th European Conference on Synthetic Aperture Radar (EUSAR '08), Friedrichshafen, Germany, June 2008.

[15] M. Weiß, O. Peters, and J. Ender, "First flight trials with ARTINO," in Proceedings of 7th European Conference on Synthetic Aperture Radar (EUSAR '08), Friedrichshafen, Germany, June 2008.

[16] J. Klare, "Digital beamforming for a 3D MIMO SARimprovements through frequency andwaveform diversity," in Proceedings of the International Geoscience and Remote Sensing Symposium (IGARSS '08), vol. 5, pp. 17-20, Boston, Mass, USA, July 2008.

[17] A. Reigber, Airborne polarimetric SAR tomography, Ph.D. dissertation, University Stuttgart, Stuttgart, Germany, 2001.

[18] J. M. Lopez-Sanchez and J. Fortuny, "3-D radar imaging using range migration techniques," IEEE Transactions on Antennas and Propagation, vol. 48, no. 5, pp. 728-737, 2000.

[19] J. Fortuny, Efficient algorithms for three-dimensional near-field synthetic aperture radar imaging, Ph.D. dissertation, University of Karlsruhe, Karlsruhe, Germany, 2001.

[20] W. Tan, W. Hong, Y. Wang, Y. Lin, and Y. Wu, "Synthetic aperture radar tomography sampling criteria and threedimensional range migration algorithm with elevation digital spotlighting," Science in China F, vol. 52, no. 1, pp. 100-114, 2009.

[21] Y. Qixiao and S. Yonghuan, Practical Mathematics Handbook, Science Press, Beijing, China, 2nd edition, 2006.

[22] W. G. Carrara, R. S. Goodman, and R. M. Majewski, Spotlight Synthetic Aperture Radar Signal Processing Algorithms, Artech House, Boston, Mass, USA, 1995. 